\title{
Birth Justice. Die Bedeutung von Intersektionalität für die Begleitung von Schwangerschaft, Geburt und früher Elternschaft
}

\author{
Christiane Winkler • Emine Babac
}

Angenommen: 14. Januar 2022

(C) Der/die Autor(en) 2022

Zusammenfassung US-amerikanische Schwarze Feminist*innen entwickelten den Begriff Birth Justice, um strukturell verankerte Ungleichheits- und Gewaltverhältnisse rund um Schwangerschaft, Geburt und frühe Elternschaft sichtbar zu machen (Oparah 2015). Birth Justice verortet sich im Kontext des Konzeptes von Reproductive Justice und ist durch eine menschenrechtsbasierte, intersektionale Analyse reproduktiver Ungleichheitsverhältnisse gekennzeichnet (Oparah 2015; Ross und Solinger 2017).

Die individuellen Erfahrungen Schwangerer* und Gebärender* werden geprägt durch die vielfältigen Wechselwirkungen zwischen Merkmalen wie geschlechtlicher Identität, Race/Ethnizität/Nationalität, Klasse, Alter, sog. Behinderung und sexueller Orientierung (ebd.). Subjektive Erfahrungen wirken sich auf Zufriedenheit mit der gesundheitlichen Versorgung aus und beeinflussen zukünftige Gesundheitsentscheidungen (Ohlbrecht 2016). Dadurch können Ungleichheiten fortgeschrieben werden.

Zufriedenstellende intersektional orientierte quantitative und qualitative Studien zu peripartalen Outcomes sowie zum Erleben der gesundheitlichen Versorgung stehen im deutschsprachigen Raum aus. Quantitative Erhebungen zu Outcomes Gebärender* mit Migrationshintergrund in Deutschland sind sehr begrenzt und kritisch zu betrachten, da sie nicht auf der Grundlage differenzierter Antidiskriminierungs-

Die Autoren haben zu gleichen Teilen zum Manuskript beigetragen.

Christiane Winkler $(\bowtie)$

Institut für Gesundheits- und Pflegewissenschaft, Martin-Luther-Universität Halle-Wittenberg,

Halle/Saale, Deutschland

E-Mail: christiane.winkler@uk-halle.de

Pörstener Str. 9, 04229 Leipzig, Deutschland

Emine Babac $(\triangle)$

Holsteinische Str. 22, 12161 Berlin, Deutschland

E-Mail: hebamme.emine@mailbox.org 
daten durchgeführt wurden. Qualitative Studien zu Erfahrungen marginalisierter/ minorisierter Personen mit peripartaler Betreuung gibt es nur sehr unzureichend in Quantität und Qualität. Ergebnisse zu Diskriminierungserfahrungen sind bisher noch nicht publiziert.

Um menschenrechtsbasierte, respektvolle Betreuung als Qualitätsmerkmal der Gesundheitsversorgung Realität werden zu lassen und gesundheitliche Ungleichheiten abzubauen, braucht es verlässliche Erhebungen zu Erfahrungen mit der peripartalen Versorgung insbesondere von marginalisierten und minorisierten Personen. Diese sollten in ihrem Design intersektional reflektiert und diskriminierungssensibel angelegt sein. Intersektionalität und ihre Auswirkung auf den Zugang und die Qualität der peripartalen Begleitung sollten in die theoretische als auch praktische Ausbildung im Gesundheitswesen aufgenommen und Bewusstsein durch Fortbildungen gefördert werden.

Schlüsselwörter Birth Justice · Reproduktive Gerechtigkeit · Intersektionalität · Schwangerschaft $\cdot$ Geburt $\cdot$ Diskriminierung

\section{The meaning of intersectionality in the provision of maternity care and early parenthood}

Abstract US Black-American feminists have developed the term Birth Justice to illustrate structural injustice and violence in the context of maternity care including pregnancy, childbirth, and the early parenthood (Oparah 2015). Birth Justice contextualises within the concept of Reproductive Justice and is marked by a human rights and intersectionality focused analysis of reproductive injustice (Oparah 2015; Ross und Solinger 2017).

Individual experiences of pregnant and birthing parents are influenced by a variety of characteristics including gender identity, race/ethnicity/citizenship, class, age, socalled disabilities, sexual orientation. Subjective experiences have an impact on the satisfaction with healthcare provision, which may influence future healthcare decisions (Ohlbrecht 2016). This in turn can lead to the continuation of disparity.

Reliable quantitative and qualitative studies including an intersectional perspective in German-speaking countries are not available yet. Furthermore, quantitative data on childbearing people with a migration background are limited and may not pass critical appraisal due to missing fundamental background information including antidiscrimination-data. In addition, qualitative research focusing on the experience of marginalised minority parents within maternity care is very limited in both number and design. Moreover, an analysis of experience of discrimination is not published yet.

For human-rights-based and respectful maternity care provision to become reality and to reduce healthcare inequities, reliable research regarding experience within maternity healthcare provision especially of marginalised minority parents is needed. However, these should reflect intersectional aspects and should include a discrimination-sensitive perspective. Furthermore, intersectionality and its impact on the access to and quality of maternity care provision should be included in both train- 
ing and ongoing post-graduation education of healthcare professionals to create and improve awareness in German-speaking countries.

Keywords Birth Justice · Reproductive Justice · Intersectionality · Pregnancy •

Childbirth · Discrimination

$\begin{array}{ll}\text { Abkürzungen } \\ \text { ABM } & \text { Alternative Birth Movement } \\ \text { ACRJ } & \text { Asian Communities for Reproductive Justice } \\ \text { BJ, BJM } & \text { Birth Justice, Birth Justice Movement } \\ \text { BIPoC } & \text { Black, Indigenous and People of Color } \\ \text { BIW*oC } & \text { Black/Indigineous Women* of Color } \\ \text { NAPW } & \text { National Advocates for Pregnant Women } \\ \text { RJ, RJM } & \text { Reproduktive Justice, Rreproductive Justice Movement } \\ \text { SES } & \text { Socioeconomic status } \\ \text { SJ } & \text { Social Justice }\end{array}$

\section{Einführung}

Kurz nachdem die 27-jährige Aktivistin und Black-Lives-Matter-Ikone Erica Garner im August 2017 ihr Kind gebar, erlitt sie einen Herzinfarkt. Sie trat als Aktivistin 2014 an die Öffentlichkeit, nachdem ihr asthmakranker Vater Eric Garner infolge einer übermäßig gewaltvollen Festnahme durch die Polizei verstorben war. Erica Garner litt unter Kardiomyopathie. Die Belastung der Schwangerschaft schwächte ihr Herz weiter. Vier Monate nach der Geburt erlitt sie einen weiteren Herzinfarkt, der sie ins Koma versetzte, aus dem sie nie wieder erwachte (Center for American Progress 2018). Auch wenn Todesfälle in jungem Alter stets tragisch sind, offenbart Erica Garners Tod ein verheerendes Problem in den Vereinigten Staaten: Schwarze Frauen* sterben drei- bis viermal häufiger im Kontext von Schwangerschaft und Geburt als weiße Frauen* (Creanga et al. 2017) und ihre Säuglinge sterben doppelt so häufig wie die Säuglinge Weißer (CDC 2016). Diese Tendenz zeigt sich unabhängig vom sozioökonomischen Status (SES) der gebärenden Person. Selbst Tennislegende Serena Williams als erfolgreiche, wohlhabende und gesunde Frau, erlitt schwere Komplikationen nach der Geburt ihrer Tochter und brachte 2018 die Debatte um Rassismus im Gesundheitswesen und insbesondere in der Geburtshilfe erneut ins Blickfeld (Center for American Progress 2018). Immer mehr Forschungsergebnisse deuten darauf hin, dass Stress aufgrund von Diskriminierungserfahrungen eine bedeutsame Rolle für maternale* und infantile Mortalität und Morbidität spielt (Center for American Progress 2018). Ob sich vergleichbare Situationen auch im deutschsprachigen Raum ereignen, ist ungewiss.

Dieser Beitrag erläutert zunächst die Bewegung für Birth Justice (BJ), die u.a. aufgrund dieser beschriebenen Ungerechtigkeiten in den USA entstand und den Rahmen für die im Anschluss zu untersuchenden Fragen bildet. BJ verortet sich im Kontext des Konzeptes von Reproductive Justice und zeichnet sich durch eine menschenrechtsbasierte, intersektionale Analyse reproduktiver Ungleichheitsverhältnisse 
aus. Vor diesem Hintergrund geht der Beitrag anschließend folgenden auf einander aufbauenden erkenntnisleitenden Fragen nach:

1. Lassen sich für rassifizierte ${ }^{1}$ Gebärende in Deutschland perinatale Ungleichheiten nachweisen?

2. Werden Erfahrungen marginalisierter/minorisierter Personen mit peripartaler Betreuung explizit in gesundheitswissenschaftlicher Forschung berücksichtigt?

3. Welche Schlussfolgerungen lassen sich aus einer intersektionalen Perspektive für Forschung und Praxis ziehen, um Ungleichheiten abzubauen und gerechte, klient*innenzentrierte und inklusive peripartale Begleitung zu fördern?

In diesem Sinne wird die Datenlage zu perinatalen Outcomes Schwarzer Gebärender in den USA sowie zur Ursachenforschung perinataler Ungleichheiten zusammengefasst. Anschließend wird der Forschungsstand zu perinatalen Ungleichheiten in Deutschland unter Gebärenden mit und ohne sogenannten Migrationshintergrund kritisch betrachtet, um zu ermitteln, ob vergleichbare Schlüsse zu ziehen sind.

Eine intersektionale Perspektive lenkt den Fokus auf all jene, die aufgrund unterschiedlichster Merkmale von der Dominanzgesellschaft bzw. -kultur abweichen und aus diesem Grund Diskriminierungserfahrungen machen können. Subjektive Perspektiven formen die Interaktionssituationen zwischen dem Gesundheitspersonal und jenen, die die gesundheitlichen Leistungen in Anspruch nehmen, und wirken sich u.a. auf die Inanspruchnahme der Leistungen und v.a. auf die Zufriedenheit mit der gesundheitlichen Versorgung aus (Ohlbrecht 2016). Daher werden anschließend qualitative europäische Erhebungen zum subjektiven Erleben der peripartalen Gesundheitsversorgung von diversen vulnerablen Gruppen identifiziert, um Forschungslücken aufzuzeigen. Abschließend sollen Anregungen für zukünftige gesundheitswissenschaftliche Forschung sowie für die Versorgungspraxis aus einer intersektionalen Perspektive gegeben werden.

\subsection{Anmerkungen über Sprache}

Die Autorinnen möchten keine Vorurteile reproduzieren, Menschen verunsichtbaren und/oder diskriminieren. Inklusive Sprache beinhaltet sowohl die Anerkennung, dass nicht alle Personen, die schwanger werden und gebären, Frauen sind, als auch die Tatsache, dass nicht alle Frauen schwanger werden. Der allgemeine Begriff Frau ist daher gleichzeitig zu eng und zu weit gefasst (Ross und Solinger 2017). Durch die Nutzung des Asterisks in diesem Beitrag wird symbolisiert, dass es sich bei Personengruppen nicht nur um zwei Geschlechter handelt. Die Begriffe Frau* und maternal* wurden um das Asterisk ergänzt, um darauf hinzuweisen, dass sich hinter dieser Kategorie multiple Geschlechtsidentitäten verbergen können. Da Gebärende und Schwangere für die Autorinnen nicht mit einer Geschlechtsidentität verbunden

\footnotetext{
1 Rassifizierung bezeichnet einen Prozess, in dem Menschen nach spezifischen Merkmalen sterotypisiert und hierarchisiert werden. Der Begriff meint, dass es sich um konstruierte Kategorien handelt, die zu realen Wirkungen (Rassismus) führen (Barskanmaz 2019).
} 
sind, wurde in diesem Artikel darauf verzichtet, wissend, dass dies unterschiedlich gehandhabt werden kann.

Rassismus wird in diesem Beitrag als ein strukturelles Phänomen verstanden. Mit der Großschreibung des Adjektivs Schwarz sowie der Beibehaltung des englischen Zusatzes of Color werden rassistische Fremdbezeichnungen abgelöst. Diese Begriffe stellen Sammelbegriffe von und für Menschen mit Rassismuserfahrungen aufgrund ethnischer Zuschreibungen dar (Kelly 2019). Es geht dabei um die Benennung von Rassismus und die Machtverhältnisse in einer mehrheitlich weißen Gesellschaft. Die Bezeichnung BIPoC (Black, Indigenous and People of Color) bezieht sich auf Schwarze, Indigene und Menschen of Color. Die Bezeichnung wei $\beta$ wird rassismuskritisch genutzt, ohne dabei explizit weiße Hautfarbe zu meinen. Vielmehr verweist der Begriff auf weiße Privilegien und entsprechende Machtverhältnisse (Barskanmaz 2019).

Im Gegensatz zu „rassistisch“ leitet sich „rassisch“ (engl. racial) von „Rasse“ ab (Barskanmaz 2019). Im Folgenden wird von rassischer statt von ,rassistischer Diskriminierung“ gesprochen, um auf „Diskriminierung aufgrund der Rasse“ aufmerksam zu machen.

\section{Methodologie}

Da diese Arbeit mehrere ineinandergreifende Themen umfasst, wurden verschiedene angepasste Suchstrategien nach dem Mixed-methods-review-Prinzip angewandt. Das Ziel dieser Recherchen war, den politisch-theoretischen Rahmen des Konzeptes von Birth Justice zu erfassen, Perinatale Outcomes Schwarzer bzw. rassifizierter Gebärender in den USA und vor allem im deutschsprachigen Raum einzuschätzen und Erfahrungen diverser vulnerabler Gruppen mit Diskriminierung während peripartaler gesundheitlicher Versorgung zu evaluieren.

Das Konzept Birth Justice wurde anhand einer theoretischen Literaturrecherche erfasst. Zum Thema Perinatale Ungerechtigkeit unter Schwarzen Schwangeren und Gebärenden wurde nach Teilnahme an einem Webinar „Understanding Systemic Racism in Maternity Care: The Voices of Black Researchers" gezielt die darin verwiesene Literatur gesichtet. Da es sich in diesem Teil v. a. um Hintergrundinformationen aus dem anglo-amerikanischen Raum handelt und der Fokus auf den deutschsprachigen Ländern liegt, wurde hier eine Scoping Review durchgeführt. Aufgrund der Suche nach konkreten Inhalten und da diese so ausreichend gedeckt waren, wurde auf eine systematische Literaturrecherche verzichtet.

Zu perinatalen Daten in Deutschland wurde eine systematische Recherche zu Geburtsoutcomes im Zusammenhang mit Migrationshintergrund durchgeführt. Da die Kategorie Migrationshintergrund meist die einzige zur Verfügung stehende Variable in quantitativen Datensätzen darstellt, um Outcomes rassifizierter bzw. kulturalisierter Personen zu bewerten, musste auf diese zurückgegriffen werden, um einen Zusammenhang zwischen Rassismus und Morbiditäts- sowie Mortalitätsraten einschätzen zu können. Dies geschah mangels Alternativen wohlwissend, dass nicht alle rassifizierten/kulturalisierten Personen einen Migrationshintergrund aufweisen, wie auch Personen mit sog. Migrationshintergrund gesellschaftlich als zur Domi- 
nanzgesellschaft zugehörig betrachtet und behandelt werden können. Da die identifizierten Veröffentlichungen allerdings zu unterschiedlich waren, um sie systematisch zu vergleichen und teilweise scheinbar die gleichen Datensätze zur Analyse genutzt wurden, werden die Ergebnisse narrativ zusammengefasst.

Um einen Überblick über qualitative Erhebungen in Europa hinsichtlich des subjektiven Erlebens der gesundheitlichen peripartalen Versorgung von spezifischen vulnerablen Gruppen zu erhalten, wurde eine Scoping Review durchgeführt. Diese Art der Literaturrecherche unter Anwendung von systematischen Suchkriterien wurde gewählt, um einen ersten Überblick über die vorhandene Evidenz in einem komplexen und heterogenen Themenfeld zu erhalten, Forschungslücken für den deutschsprachigen Raum auszuweisen und Empfehlungen für zukünftige Forschungen zu unterbreiten. Bewertung, Auswertung und Diskussion erfolgt folglich nicht systematisch durch entsprechende Kategorienbildung, sondern narrativ und deskriptiv (Grant und Booth 2009; von Elm 2019).

Die Suche erfolgte in PubMed, CINAHL und Cochrane. Um möglichst umfassende Ergebnisse hinsichtlich besonders vulnerabler Gruppen zu erhalten, wurden diese in einer zusätzlichen Suche erfasst (Tab. 1 Literaturrecherche - Suchstrategie).

Eingeschlossen wurden qualitative Studien und Studien mit Mixed-Methods-Ansätzen der letzten 20 Jahre, deren qualitative Datenerhebung sich auf die Erfahrungen der Personen bezieht, die Gesundheitsdienstleistungen im Kontext von Schwangerschaft, Geburt und Wochenbett in Anspruch nehmen. Studien, die sich ausschließlich mit der Perspektive der Gesundheitsfachkräfte befassten, wurden ausgeschlossen sowie Studien, deren Abstracts nicht ausreichende Informationen boten.

Insgesamt konnten 55 in Europa durchgeführte Studien identifiziert werden, die sich auf diverse Klient*innengruppen beziehen. Davon wurden lediglich zwei im deutschsprachigen Raum durchgeführt, eine in Österreich im Kontext sog. Behinderung (Schildberger et al. 2017) und eine weitere in der deutschsprachigen Schweiz im Kontext von Migration (Origlia Ikhilor et al. 2019). Der insgesamt größte Teil der Arbeiten bezieht sich auf Erfahrungen von Personen im Kontext von Migration und auf Erfahrungen von Angehörigen ethnischer Minderheiten $(n=32)$. Acht Studien befassen sich mit Erfahrungen von Menschen aus dem LGBTIQ+ Spektrum. Sechs Studien behandeln Erfahrungen von Schwangeren, die behindert werden und/

Tab. 1 Literaturrecherche - Suchstrategie

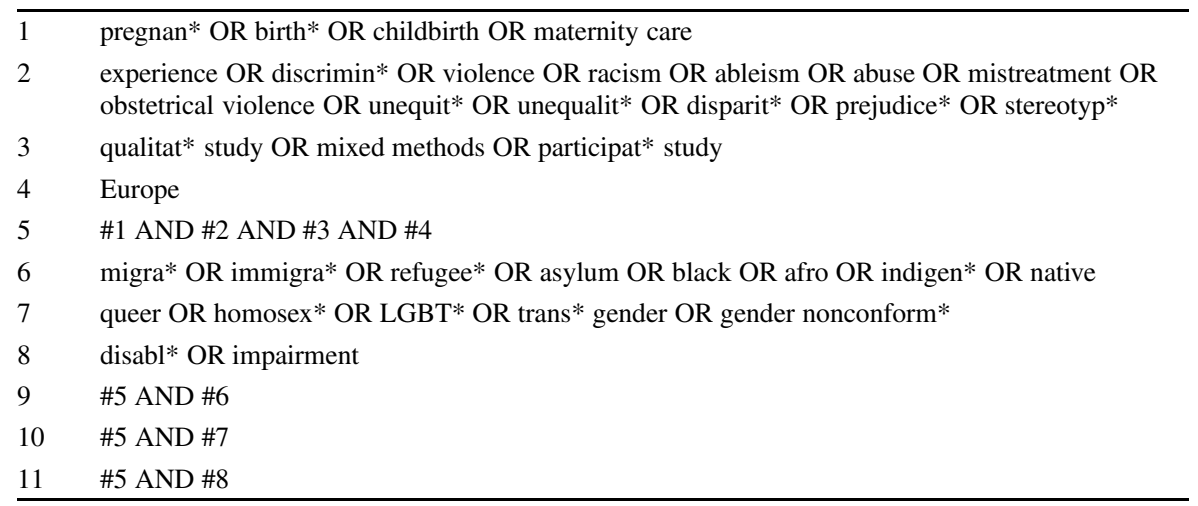


oder mit sog. Lernschwierigkeiten. Zwei weitere fokussieren die Erfahrungen von fettleibigen Schwangeren. Die restlichen sieben beziehen sich entweder auf die Erfahrungen von Frauen* im Allgemeinen $(n=3)$ oder konzentrieren sich auf jene mit außergewöhnlichen Umständen, wie Menschenhandel $(n=1)$, positivem HIV-Test in der Schwangerschaft $(n=1)$, sexualisierte Gewalterfahrung in der Kindheit $(n=1)$ und Gewalterfahrung im Gesundheitswesen $(n=1)$. Eine zusätzliche Studie beschäftigte sich im Rahmen von Migration auch mit weiblicher Genitalverstümmlung. Die meisten Studien wurden in Großbritannien $(n=24)$ und Skandinavien $(n=19)$ durchgeführt. Diese werden anschließend erläutert und diskutiert.

\section{Reproduktive Gerechtigkeit und Geburt}

\subsection{Birth Justice}

Auch wenn das Schlagwort Reproductive Justice (RJ) inzwischen in hiesigen feministischen Diskursen vereinzelt auftaucht (Kitchen Politics 2021), ist der Begriff BJ noch weitestgehend unbekannt. Lange bevor der Begriff in den USA geprägt wurde, ebneten Schwarze Frauen* durch ihren Widerstand sowohl gegen die Misshandlung Schwarzer Schwangerer während der Sklaverei als auch gegen die Unterdrückung Schwarzer Geburtspraktiken und Familienstrukturen den Weg für die heutigen Aktivist*innen für BJ (Monroe 2015). Die individuellen Erfahrungen Schwarzer Frauen* während Schwangerschaft, Geburt und Wochenbett sind nicht nur von Gewalt und Zwang durch patriarchale Institutionen geprägt, sondern auch durch die vielfältigen Wechselwirkungen zwischen geschlechtlicher Identität, „Rasse“/Ethnizität/Nation, Klasse, Alter, Befähigung bzw. sog. Behinderung und sexueller Orientierung (Oparah 2015). Seit ca. 15 Jahren wurden zahlreiche US-amerikanische Organisationen aktiv, wie z. B. Black Women Birthing Justice, Black Mamas Matter Alliance oder Southern Birth Justice Network, welche die Erfahrungen von Black/Indigineous Women* of Color (BIW*oC), trans* und gender-nonconforming Eltern fokussieren. Sie versuchen, sie zu befähigen, für respektvolle Geburtshilfe und gegen systemische Diskriminierung aktiv zu werden (Oparah 2015). Da sich die Bewegung für BJ im Kontext von RJ verortet, folgt zunächst eine nähere Betrachtung des Reproductive Justice Movements (RJM).

\subsection{Reproductive Justice}

Der Begriff RJ wurde in den 90ern durch Schwarze Feminist*innen und Feminist*innen of Color in den USA geprägt. Motiviert durch die Frustration über ein ausschließlich individualistisches Verständnis von Autonomie, wie es im Mainstream der Pro-Choice-Bewegung vorherrschte, entstand ein Bündnis, um das Konzept der reproduktiven Rechte zu erweitern und durch einen menschenrechtsbasierten Ansatz neu zu definieren (Price 2020). Das RJ Movement (RJM) schließt durch diesen erweiterten Ansatz mehr Schwarze, Frauen* of Color und weitere marginalisierte Gruppen ein und führt dadurch zur verstärkten Mobilisation von Aktivist*innen für reproduktive Rechte (Price 2020). Das vorrangige Ziel der Bewegung ist, sich 
über den engen Fokus auf das Recht auf Schwangerschaftsabbruch hinauszubewegen. Die in Oakland ansässige Organisation Asian Communities for Reproductive Justice (ACRJ) definiert RJ folgendermaßen:

We believe reproductive justice is the complete physical, mental, spiritual, political, economic, and social well-being of women and girls, and will be achieved when women and girls have the economic, social and political power and resources to make healthy decisions about our bodies, sexuality and reproduction for ourselves, our families and our communities in all areas of our lives. (ACRJ 2005, S. 1).

Loretta Ross, Mitgründerin der Organisation Sister Song und bekannteste Aktivistin des RJM stellt drei primäre Werte des Konzeptes heraus: 1. das Recht, kein Kind zu bekommen, d.h. das Recht eine Schwangerschaft zu verhindern oder abzubrechen, 2. das Recht Kinder zu bekommen, also schwanger zu werden, und 3. das Recht, Kinder in einer sicheren und gesunden Umgebung aufzuziehen. Darüber hinaus fordert das Konzept sexuelle Autonomie und geschlechtliche Selbstbestimmung für jeden Menschen (Ross und Solinger 2017). Menschen, die schwanger werden können, müsse es möglich sein, diese Rechte frei von Zwang, Unterdrückung und Diskriminierung zu leben. Das Konzept RJ zeichnet sich somit durch eine Erweiterung des Paradigmas der sexuellen und reproduktiven Gesundheit und Rechte durch eine intersektionale Analyse reproduktiver Unterdrückungs- und Diskriminierungsmechanismen aus (Price 2020). Die ACRJ differenziert die Konzepte von reproduktiver Gesundheit, reproduktiver Rechte und reproduktiver Gerechtigkeit und sieht in den drei, sich ergänzenden Konzepten in der Summe einen umfassenden Ansatz zur Auflösung reproduktiver Unterdrückung (ACRJ 2005). RJ basiert auf der Annahme, dass zwar jeder Mensch die gleichen Menschenrechte besitzt, Unterdrückungsund Diskriminierungserfahrungen sich jedoch auf vielfältige Weise unterscheiden. Die meisten Menschen machen im Laufe ihres Lebens Erfahrungen interpersoneller und/oder institutioneller Diskriminierung aufgrund persönlicher Merkmale wie „Rasse“/Ethnie/Nation, Klasse, Alter, Religion, geschlechtlicher Identität, sexueller Orientierung, sog. Behinderung.

Das Konzept RJ verbindet reproduktive Gesundheit und reproduktive Rechte mit anderen Themen im Kontext von Social Justice ${ }^{2}$ (SJ) wie z. B. ökonomische Gerechtigkeit, Bildung, Gentrifizierung, Migrant*innenrechte u. a. und geht damit weit über die Pro-Choice/Pro-Life-Debatte hinaus. RJ betrachtet, wie sich diese Themen überschneiden und welche Auswirkungen sie an verschiedenen Schnittpunkten auf das Leben und die Körper von Menschen haben, die schwanger werden können (Ross und Solinger 2017). So haben Organisationen für RJ begonnen, das Zusammenwirken von Merkmalen wie „Rasse“, SES und Geschlechtsidentität zu untersuchen. Dieses spezifische Zusammenspiel wirkt sich auf den Zugang zu reproduktiver Ge-

\footnotetext{
2 Unter SJ verstehen die Autorinnen ein charakteristisches Konzept von Gerechtigkeit, das simultan Verteilungs-, Anerkennungs-, Befähigungs- und Verwirklichungsgerechtigkeit in den Fokus nimmt. Da das Konzept des SJ damit den gebräuchlichen deutschsprachigen Begriff der sozialen Gerechtigkeit übersteigt, verwenden die Autorinnen auch für dieses Konzept den englischen Begriff (Czollek et al. 2019).
} 
sundheitsversorgung aus und generiert u.a. passive Eugenik, die sich z.B. gegen trans* und gender-nonconforming BIPoC richtet (Nixon 2013; Oparah 2015). RJ betrachtet reproduktive Unterdrückung als Ergebnis der Überschneidung bzw. als Schnittmengen mehrerer Diskriminierungsformen und ist damit inhärent mit dem Kampf für SJ und für die Menschenrechte verbunden (ACRJ 2005).

\subsection{Die Merkmale der Bewegung für Birth Justice}

Im Jahr 2010 rief die National Advocates for Pregnant Women (NAPW) andere Organisationen für RJ dazu auf, ihre Agenda, um die geburtshilfliche Versorgung für gebärende Personen zu erweitern (NAPW 2010). Während die NAPW in diesem Aufruf der Bewegung für RJ großen Respekt zollt, macht sie gleichzeitig Widersprüche sichtbar, die entstehen, wenn das Menschenrecht auf eine sichere und respektvolle Geburtserfahrung nicht als zentraler Teil der Agenda für RJ betrachtet wird. RJ-Aktivist*innen kämpften zwar für den ungehinderten Zugang zu Abtreibungen und sicheren Verhütungsmitteln für $\mathrm{BIW}^{*} \mathrm{OC}$ und Frauen* mit niedrigem SES, gleichzeitig träten sie jedoch selten für das Recht Gebärender auf die freie Wahl des Geburtsortes, auf vaginale Geburten nach einem Kaiserschnitt oder für flächendeckende Hebammenbetreuung ein (NAPW 2010; Oparah 2015). „They put the racially targeted sterilization of women of color on the radar of those concerned with reproductive rights but say little about epidemic rates of C-sections, which are disproportionately performed on African American women“ (Oparah 2015, S. 7). Infolgedessen bliebe das Missverständnis unwidersprochen, dass vaginale und auBerklinische Geburten sowie Hebammenbetreuung und Begleitung durch Doulas „Luxusanliegen“ weißer $\mathrm{Cis}^{3}$-Frauen der Mittelschicht seien und somit das aktivistische Potenzial Schwangerer und Eltern of Color, die von Ungerechtigkeiten im Kontext von Schwangerschaft, Geburt und Wochenbett betroffen sind, weitgehend ungenutzt bliebe (Oparah 2015).

Kimala Price untersuchte 2010 das RJM in den USA (Price 2020). Sie analysierte sowohl Interviews, die sie vorwiegend mit den Gründer*innen der Bewegung für RJ führte, als auch Dokumente wie Webseiten, Standpunktpapiere, Newsletter u.v.m. Im Ergebnis der Analyse identifizierte sie folgende drei Narrative: 1. Origin Stories, 2. Opposition Stories und 3. Cautionary Tales, welche auch zum Verständnis der Bewegung von BJ beitragen (Price 2020).

\subsubsection{Origin Stories - Entstehungsgeschichten}

In der Entstehungsphase einer sozialen Bewegung ist es wesentlich, eine Identität der Bewegung zu konstruieren, welche nicht nur ihr Selbstverständnis reflektiert, sondern sie auch als tragfähigen und effektiven politischen Mitstreiter etabliert. Dieser Prozess der Identitätskonstruierung beinhaltet laut Price (2020) das wiederholte Erzählen der Entstehungsgeschichte und der Absichten (Price 2020).

\footnotetext{
${ }^{3}$ Cis (diesseits) ist als Begriff das Gegenstück zu trans (jenseitig). In diesem Kontext bezeichnet es Menschen, deren Geschlechtsidentität mit dem ihnen bei der Geburt zugewiesenen Geschlecht übereinstimmt.
} 
Im Folgenden ein Beispiel der BJ Aktivistin Shafia Monroe aus der Einleitung des Sammelbandes „Birthing Justice: Black Women, Pregnancy, and Childbirth“ (Oparah und Bonaparte 2015): „Long before the term „birth justice“ was coined, black women used traditional childbearing knowledge, oral histories, human rights organizing, and policy work in their efforts to end inequities in maternal, infant, and child health. Building on resistance to the abuse of pregnant black women during slavery and to the dismantling of our birth traditions and family structures, our foremothers paved the way for today's birth justice activism. [...] Our present day birth justice movement honors its ancestors. [...] Fortunately, through the efforts of the birth justice movement, women of color are entering midwifery training and becoming midwives at record numbers; [...] Birth justice is on the rise. Women and trans/gender nonconforming birthing parents are mobilizing internationally to reclaim birth [...] and to demand their human right to birth and breastfeed their infants according to their own traditions. [...] It is up to all of us to ensure that this movement toward birth justice continues." (Monroe 2015, S. 1).

Für die Aktivist*innen liegt der Zweck in der kontinuierlichen Betonung dieser und ähnlicher Aussagen v. a. darin, an die Gründer*innen der Bewegung zu erinnern, sie zu ehren und anhand der Beispiele bisheriger Kämpfe und Errungenschaften junge Frauen* of Color aufzuklären und sie dazu zu inspirieren, Nachfolger*innen in der Anführung der Bewegung zu werden (Price 2020).

\subsubsection{Opposition Stories - Gegenerzählungen}

Zur Bildung einer eigenen Identität definiert sich eine Bewegung nicht ausschließlich darüber für was sie steht, sondern auch darüber, wofür sie nicht steht. Price nennt diese Narrative Opposition Stories (Gegenerzählungen) (Price 2020).

Die Forderungen des BJM ähneln denen des in den 60er- und 70er-Jahren entstandenen Alternative Birth Movements (ABM) (Oparah 2015). Dennoch sieht die Bewegung für BJ eklatante Differenzen mit dem ABM, die sie als Ursache dafür betrachtet, dass es dem ABM nicht gelungen ist, Frauen* of Color und andere marginalisierte Gruppen zu mobilisieren und für ihre Anliegen zu gewinnen. Der Diskurs um die ,natürliche“ Geburt, beschreibt diese als eine Erfahrung, die allen Frauen (sic!) vom patriarchalen, medizinischen Establishment gestohlen worden sei, um deren Körper zu kontrollieren und kommerzialisieren (Oparah 2015). Dies ignoriert jedoch die rassistischen und klassistischen Ursprünge der modernen Geburtshilfe, welche sich bis in die gegenwärtigen Versorgungssysteme im Kontext von Schwangerschaft, Geburt und Wochenbett fortschreiben. Zwar betreuten traditionelle Hebammen* in ihren Communities BIW*oC und arme weiße Frauen* v. a. in ländlichen Gebieten weiterhin während der Geburt zu Hause, dies sei jedoch kein Akt der Selbstbestimmung, sondern die Folge der Ablehnung und Vernachlässigung marginalisierter Gruppen durch die weiße Ärzteschaft und die Gesellschaft im Ganzen (Oparah 2015). „For too many black women and their infants, denied adequate health care and nutrition for a livetime, ,natural“ childbirth meant a preventable death" (Oparah 2015, S. 11). Oparah (2015) führt den Erfolg des ABM zum Teil auf deren Ausrichtung auf hegemoniale Vorstellungen von „Rasse“, Mutterschaft und neoliberales Konsumdenken zurück. Gleichzeitig moniert sie: „Reducing birth 
justice to the right to shop has negative consequences, however, particularly for poor women, women with disabilities, trans/gender nonconforming people, and others who are constructed as recipients and dependents rather than consumer-citizens“" (Oparah, S. 14).

Wie Price (2020) in Bezug auf das Verhältnis von RJ zum Pro-Choice Movement ausführt, ist auch die Bewegung für BJ weder als Gegenbewegung zum ABM noch als Untergruppe dieser zu verstehen. Vielmehr ist es eine eigene Bewegung, die sich dadurch auszeichnet, intersektionale Analysen ins Zentrum ihrer politischen Agenda zu rücken. Die Bewegung für BJ bildet ihre Identität unter anderem durch Abgrenzung vom ABM, indem dessen Konzept der Selbstbestimmung kritisiert und gleichzeitig ein alternatives Konzept angeboten wird (Oparah 2015).

\subsubsection{Cautionary Tales - Warnungen der Geschichte}

Unter sogenannten ,cautionary tales“ versteht Price warnende und moralische Botschaften, die aus konkreten (historischen) Ereignissen abgeleitet werden (Price 2020). Eugenische Praktiken sowie medizinische Experimente stehen dabei im Mittelpunkt der Diskurse. Dabei liegt der Fokus im RJM v.a. auf der kritischen Analyse der (Dis-)Kontinuitäten eugenischer Praktiken wie Zwangssterilisierungen und Maßnahmen zur Geburtenkontrolle (Ross und Solinger 2017). Gynäkologische und geburtshilfliche Experimente an Schwarzen schwangeren und gebärenden Personen bzw. der erzwungene Zugriff auf ihre Körper für den Zweck des medizinischen Fortschritts bilden die Inhalte der ,cautionary tales“ des BJM. In Anlehnung an den von Washington (2006) geprägten Begriff der ,medical apartheid“ führt Oparah (2015) den Begriff der „obstetrical apartheid“ ein. So wurden für die Entwicklung sogenannter medizinischer Fortschritte versklavte Schwarze Frauen* für unzählige Experimente gefoltert, sodass es ihre Gesundheit, ihr Leben und das ihrer Kinder forderte. Washington nennt diesen Prozess und sein fortwährendes Vermächtnis gesundheitlicher Ungerechtigkeiten medizinische Apartheid. „As coerced obstetric research subjects, black women have suffered intolerable pain, disability, and even death, as well as the loss of their infants, so that white female patients could benefit from the perfected medical procedures“ (Oparah 2015, S. 10). Im Anschluss an dieses historische Erbe wirkt es infam, die medikalisierte Geburt lediglich als patriarchale Erfindung männlicher Ärzte darzustellen und die rassistischen und klassistischen Ursprünge der Geburtshilfe auszublenden. Die Entwicklung der medikalisierten Geburt ist laut Oparah (2015) diesem System der medizinischen Apartheid zu verdanken. Der Prozess, gekennzeichnet durch das Zusammenwirken patriarchalen medizinischen Heldentums, rassifizierter medizinischer Gewalt, ökonomischer Ausbeutung und rücksichtsloser Missachtung der Gesundheit Schwarzer Frauen* wird daher von ihr als ,geburtshilfliche Apartheid“ bezeichnet.

Diese drei Gruppen an Narrativen dominieren den Prozess der Identitätsbildung sowohl des RJM als auch des BJM. Sie dienen jedoch nicht nur der Definition der Bewegungen in ihrer inhaltlichen Ausrichtung, sondern auch der Abgrenzung vom Mainstream der Pro-Choice-Bewegung sowie des ABM und sind im Vergleich zu diesen durch eine holistische, intersektionale Agenda gekennzeichnet (Price 2020). 


\section{Perinatale Ungleichheiten}

\section{1 „Rasse“ als eigenständiger Risikofaktor für Gebärende und ihre Kinder in den USA}

Schwarze Frauen* in den USA erfahren unverhältnismäßig schlechte perinatale Gesundheitsergebnisse, wie z.B. hohe Raten schwangerschaftsbedingter Todesfälle und schwere maternale* Morbidität (National Partnership for Women und Families 2018). Die schwangerschaftsbedingte Mortalitätsrate Schwarzer Frauen* lag in den Jahren 2011-2013 3,4-mal höher als die Mortalitätsrate weißer Frauen* (Creanga et al. 2017). Im Zeitraum 2011-2016 betrug die schwangerschaftsbedingte Mortalitätsrate unter Schwarzen Frauen* 42,2 pro 100.000 Geburten im Vergleich zu 13,0 unter weißen Frauen* (CDC 2016). Die gesundheitliche Ungleichheit hinsichtlich der maternalen* Mortalitätsrate und schwerer maternaler* Morbidität betrifft alle Schwarzen Frauen* unabhängig ihres SES (Black Mamas Matter Alliance \& Center for Reproductive Rights 2016).

Auch die Säuglingssterblichkeit ist unter der Schwarzen Bevölkerung doppelt so hoch wie für Weiße (CDC 2016). Im Jahr 2016 verstarben 11,4 Schwarze Säuglinge pro 1000 Lebendgeburten im Vergleich zu 4,9 weißen Säuglingen (CDC 2016). Ähnlich verhält es sich mit der Frühgeburtenrate unter Schwarzen Frauen* im Vergleich zu Weißen. So lag die Frühgeburtenrate unter Schwarzen Gebärenden (14\%) um etwa $50 \%$ höher als unter Weißen (9\%) (CDC 2016). Als Ursachen der Säuglingssterblichkeit gelten genetische Defekte, Frühgeburtlichkeit, niedriges Geburtsgewicht, Unfälle und der plötzliche Säuglingsstod. Aktuell wurde Rassismus als weitere Ursache bestimmt: implizite Voreingenommenheit bzw. unbewusste Vorurteile und die Auswirkungen von strukturellem Rassismus tragen ebenfalls zu den höheren Frühgeburtenraten Schwarzer Gebärender bei (Monroe 2020).

Die höhere Prävalenz von Risikofaktoren Schwarzer Gebärender reicht nicht aus, um die perinatalen Ungleichheiten zu erklären. Dazu gehören niedriger SES, eingeschränkter Zugang zu Schwangerschaftsversorgung, allgemein schlechtere physische und psychische Grundvoraussetzungen und vorbestehende Erkrankungen. Obwohl Schwarze Frauen* mit größerer Wahrscheinlichkeit von diesen miteinander in Beziehung stehenden Risikofaktoren betroffen sind als Weiße, zeigt die Forschung, dass dies als alleinige Erklärung für die perinatalen Ungleichheiten zwischen Schwarzen und weißen Frauen* nicht ausreicht. Vielmehr sind diese Ungleichheiten auf rassische und geschlechtsbezogene Diskriminierung zurückzuführen, denen Schwarze Frauen* zeit ihres Lebens ausgesetzt sind (Lu et al. 2010; Center for American Progress 2018). Die „Rasse“ selbst stellt einen Risikofaktor dar, der noch nicht vollständig erforscht ist. Als eine Erklärungsmöglichkeit werden die kumulativen Auswirkungen von systemischem Rassismus auf die maternale* und infantile Gesundheit anhand der Life-Course-Perspective (Lu und Halfon 2003) diskutiert. Diese besagt, dass struktureller Rassismus soziale und ökonomische Kräfte erzeugt, welche Schwarze und weiße Frauen* auf unterschiedliche Lebenswege führen, mit langfristigen Folgen für ihre eigene Gesundheit und die ihrer zukünftigen Kinder. Die Erfahrungen struktureller rassischer Diskriminierung - nicht die „Rasse“ selbst - be- 
einträchtigen die Gesundheit Schwarzer Frauen*, People of Color und ihrer Kinder (Center for American Progress 2018).

\subsection{Perinatale Ungleichheiten in Deutschland unter Gebärenden mit und ohne sogenannten Migrationshintergrund}

Im Folgenden soll untersucht werden, ob sich auch für rassifizierte/kulturalisierte Gebärende in Deutschland perinatale Ungleichheiten nachweisen lassen. Da in Deutschland Angaben zur „Rasse“/Ethnizität nicht vollständig in amtlichen Statistiken erhoben werden und somit die Kategorie Migrationshintergrund meist die einzige zur Verfügung stehende Variable in quantitativen Datensätzen darstellt, musste auf diese zurückgegriffen werden. Durch die Recherche zum Thema gesundheitlicher Ergebnisse in Schwangerschaft und Geburtshilfe unter Migrant*innen konnten 21 Studien identifiziert werden. Im Vergleich zur existierenden Studienlage in den USA erscheint die Datenlage in Deutschland sehr dürftig. Die Themen dieser Studien ähneln sich und beziehen sich auf geburtshilfliche Variablen wie Frühgeburtlichkeit, intrauterine Mortalität, Sectioraten und Epiduralanästhesie-Raten (PDA).

Frühgeburtlichkeit, Stillgeburten ${ }^{4}$ und ein geringes Geburtsgewicht des Neugeborenen werden häufig mit einer mangelnden Gesundheitsversorgung oder mangelndem Zugang in Verbindung gebracht (Reime et al. 2006; Reeske et al. 2011; Berger 2012). Daher untersuchen quantitative Studien diese Aspekte im Zusammenhang mit dem sogenannten Migrationshintergrund der Gebärenden. Sie versuchen dabei festzustellen, ob die Raten unter Schwangeren mit Migrationshintergrund anders sind als bei jenen ohne, um somit Rückschlüsse auf die vermeintliche Qualität der gesundheitlichen Versorgung ziehen zu können. So stellte Berger (2012) fest, dass die Frühgeburtsrate unter Gebärenden mit Migrationshintergrund tendenziell niedriger ist als bei jenen ohne. Vielmehr zeigte sich ein Zusammenhang mit sozialer Chancenungleichheit und einem niedrigen SES. Diese Ergebnisse werden von anderen Studien untermauert, die ebenfalls geringere Frühgeburtsraten unter Gebärenden mit Migrationshintergrund feststellten (Spallek et al. 2014; David et al. 2017; Zolitschka et al. 2019), während Hofen-Hohloch (2015) keine Unterschiede fand.

Die Ergebnisse in Bezug auf Stillgeburten sind unterschiedlich. Während einige Forscher*innen (Reeske et al. 2011) eine höhere Rate unter Migrant*innen mit unterschiedlicher Ausprägung abhängig von der Herkunft feststellten, fanden andere, dass sich im zeitlichen Verlauf das Verhältnis umkehrte (Spallek et al. 2014). Die Beurteilung ist allerdings schwierig, da die Stillgeburtsrate bei Gebärenden mit deutschem Hintergrund zwar kontinuierlich mit geringen Schwankungen sinkt, bei jenen mit Migrationshintergrund jedoch von Jahr zu Jahr deutlich schwankt (Razum et al. 1999). Zu bemängeln ist auch die Einschätzung der Herkunft, da in den Studien nicht nachzuvollziehen ist, wie der Migrationshintergrund der Gebärenden festgestellt wurde.

\footnotetext{
4 Stillgeburt in diesem Beitrag meint jedes Neugeborene, das potenziell lebensfähig hätte sein können, aber ohne Lebenszeichen geboren wurde.
} 
Auch in Bezug auf Neugeborene mit geringem Geburtsgewicht kann man in Deutschland keine Assoziation zwischen dem Migrationsstatus und dem neonatalen Geburtsgewicht ableiten. Darüber hinaus ist ein geringes Geburtsgewicht von den sozialen Umständen der Schwangeren abhängig (Reime et al. 2006; Zolitschka et al. 2019). Ähnliche Ergebnisse finden sich hinsichtlich erhöhter Sectioraten und -indikationen (David et al. 2018; Miani et al. 2020). Es konnte keine Korrelation zwischen Kaiserschnitten und Migrationsstatus festgestellt werden. Vielmehr wurde eine Verbindung mit dem sozialen Status ersichtlich. Die Datenlage legt sogar nahe, dass Gebärende mit Migrationshintergrund eher seltener per Sectio gebären (David et al. 2006; Hofen-Hohloch 2015; Miani et al. 2020).

In einigen Studien werden die besseren Ergebnisse Gebärender mit Migrationshintergrund hervorgehoben. So wird z. B. darauf hingewiesen, dass Frauen* mit Migrationshintergrund nicht nur seltener per Sectio Caesarea gebären, sondern auch weniger oft eine PDA erhalten und ihre Kinder seltener auf die Neugeborenen-Intensiv-Station verlegt werden (David et al. 2006, 2017, 2018; Hofen-Hohloch 2015; Boxall et al. 2018). Allerdings stellten Hofen-Hohloch (2015) und David et al. (2014) fest, dass vor allem Schwangere türkischer Abstammung häufiger prä- und postpartal anämisch sind. In einer Gruppe von vietnamesischen Gebärenden stellten Boxall et al. (2018) fest, dass diese eine höhere Wahrscheinlichkeit für Dammrisse dritten und vierten Grades aufweisen.

Zusammenfassend lässt sich feststellen, dass die Studienlage hinsichtlich des Erkenntnisinteresses wenig aussagekräftig ist, insbesondere aufgrund der bereits benannten Problematik der Kategorie „Migrationshintergrund“. Nicht alle rassifizierten/kulturalisierten Personen haben einen Migrationshintergrund und auch Personen mit sogenanntem Migrationshintergrund können gesellschaftlich als zur Dominanzgesellschaft zugehörig betrachtet und behandelt werden. Je nach verwendetem Datensatz liegen unterschiedliche Definitionen des sogenannten Migrationshintergrundes vor. Im Zuge der Perinataldaten-Erhebung hängt die Kategorisierung beispielsweise von der individuellen Einschätzung derjenigen $a b$, die die Daten erheben. Anders als in den USA werden die Folgen von Ungerechtigkeit, Diskriminierung und Rassismus in der Geburtshilfe in Deutschland somit nicht in den Ergebnissen existierender quantitativer Studien offensichtlich. Ob die Annahme, dass die Konsequenzen von Ungerechtigkeit und Diskriminierung hierzulande weniger gravierend sind, berechtig ist, bleibt vorerst unklar. Daher soll anschließend die qualitative Studienlage zu Erfahrungen mit der gesundheitlichen Begleitung von Schwangerschaft, Geburt und früher Elternschaft evaluiert werden.

\subsection{Qualitative Veröffentlichungen im Kontext von Birth Justice}

In den USA zeigen sich in den vergangenen Jahren vielversprechende Entwicklungen hinsichtlich der Bemühungen um Sensibilisierung für BJ. Einige Bundesstaaten ${ }^{5}$ haben kooperative Forschungsgruppen aus Entscheidungsträger*innen, Aktivist*innen

\footnotetext{
5 Z.B. Kalifornien und North Carolina (vgl. https://www.cmqcc.org/; https://medicaid.ncdhhs.gov/ providers/programs-services/family-planning-and-maternity/pregnancy-medical-home. Zugriff: 06.04. 2021).
} 
und Forschenden eingerichtet mit dem Ziel, die Gesundheitsergebnisse von schwangeren und gebärenden Personen zu verbessern. Zunächst lag der Schwerpunkt der gesundheitswissenschaftlichen Forschung in den USA in der epidemiologischen Erforschung des Zusammenhangs zwischen Diskriminierung und gesundheitlicher Ungleichheit (Krieger 2014). In den letzten Jahren ist jedoch eine stärkere Anwendung von qualitativen Methoden und damit eine Hinwendung zur Subjektperspektive, der Analyse von Interaktionssituationen mit Gesundheitsfachkräften und dem Aufzeigen struktureller Probleme zu beobachten (Hoffkling et al. 2017; Altman et al. 2019; Canty 2020; Franck et al. 2020). Subjektive Perspektiven formen die Interaktionssituationen zwischen dem Gesundheitspersonal und jenen, die die gesundheitlichen Leistungen in Anspruch nehmen, und wirken sich u. a. auf die Inanspruchnahme der Leistungen und v. a. auf die Zufriedenheit mit der gesundheitlichen Versorgung aus (Ohlbrecht 2016). Um das Menschenrecht auf respektvolle Betreuung in der perinatalen Gesundheitsversorgung als Qualitätsmerkmal Realität werden zu lassen, braucht es verlässliche Erhebungen zu Erfahrungen von Respektlosigkeit und Gewalt $^{6}$ in der perinatalen Versorgung insbesondere von vulnerablen und von Diskriminierung betroffenen Personen(gruppen).

Die Datenlage der bisherigen Veröffentlichungen in Europa ist begrenzt. Drei Studien, die sich mit Erfahrungen im Kontext von Migration und ethnischen Minderheiten unter expliziter Berücksichtigung intersektionaler Aspekte beschäftigten, konnten identifiziert werden (Robertson 2015; Watson und Downe 2017; LeMasters et al. 2019). LeMasters et al. (2019) und Watson und Downe (2017) fokussieren sich auf die Erfahrungen von Rom*nja. Letztere ist die Einzige, die explizit strukturelle Diskriminierungserfahrungen ins Zentrum der Erhebung stellt und entsprechend den Titel der Studie formuliert. Auch in allen anderen Studien tauchen Erfahrungen von Gewalt nach der Typologie von Bohren et al. (2015) auf. Die meisten verweisen auf Diskriminierungserfahrungen aufgrund der ethnischen Herkunft und des SES. In diesem Kontext finden sich Schlagworte wie (unbewusste) Vorurteile (z. B. Ahrne et al. 2019; Degrie et al. 2020), stereotypisierende Annahmen über den kulturellen Hintergrund (z. B. Garnweidner-Holme et al. 2017; Firdous et al. 2020), rassistische Erfahrungen (z. B. Bollini et al. 2007; Janevic et al. 2011) und respektloses, ignorierendes und verunsicherndes Verhalten der Fachkräfte den Frauen* gegenüber (z. B. Robertson 2015; Fair et al. 2020). Ungenügende Kommunikation und Beziehungsgestaltung zwischen den Fachkräften und Klient*innen wie ineffektive oder schlechte Kommunikation aufgrund von Sprachbarrieren werden gehäuft benannt (z. B. Carlsson et al. 2019; Fair et al. 2020). Des Weiteren tauchen strukturelle Barrieren wie Zugangsbarrieren zur perinatalen Gesundheitsversorgung und ein allgemeiner Mangel an Informationen hinsichtlich zustehender Versorgungsmöglichkeiten immer wieder auf (z. B. Watson und Downe 2017; Schmidt et al. 2018; Fair et al. 2020). Darüber hinaus wird die Bedeutung respektvoller und empathischer, kontinuierlicher sowie

\footnotetext{
${ }^{6}$ Bohren et al. (2015) fanden auf Grundlage eines systematischen Reviews zahlreiche Formen von Gewalt in der Geburtshilfe weltweit. Die entwickelte Typologie unterscheidet körperliche, sexualisierte und verbale Gewalt, Stigmatisierung und Diskriminierung, das Nichteinhalten professioneller Standards, ungenügende Kommunikation und Beziehungsgestaltung sowie institutionelle Bedingungen und Beschränkungen im jeweiligen Gesundheitssystem. Sie verweisen damit sowohl auf interindividuelle als auch auf strukturelle Formen der Gewalt. (Bohren et al. 2015; Limmer et al. 2020).
} 
traumasensibler medizinischer und psychosozialer Betreuung hervorgehoben (z. B. Carlsson et al. 2019; Fair et al. 2020; Utne et al. 2020). Zahlreiche Studien schlussfolgern, dass es essenziell ist, das Fachpersonal zu kulturell-sensibler Betreuung zu befähigen (z. B. Schmidt et al. 2018; Fair et al. 2020; Utne et al. 2020).

Selbstverständlich haben auch Menschen mit körperlicher Beeinträchtigung und Lernschwierigkeiten das Recht, sich fortzupflanzen und eine Familie zu gründen. Studien zeigen, dass Frauen* mit körperlicher Beeinträchtigung und/oder Lernschwierigkeiten einen besonderen Wunsch nach Familiengründung hegen, da er für die Betroffenen oft für Normalität und gesellschaftliche Zugehörigkeit steht. Gerade für sie sind die strukturellen Barrieren zur Verwirklichung des Kinderwunsches groß (Mertens 2016). Im Kontext der perinatalen Gesundheitsversorgung fanden sich lediglich sechs qualitative Studien, die sich mit den Erfahrungen von Menschen mit sogenannten Behinderungen oder Lernschwierigkeiten befassten. Davon wurden drei in Großbritannien (Bradbury-Jones et al. 2015; Malouf et al. 2017; Hall et al. 2018) durchgeführt und je eine in Irland (Walsh-Gallagher et al. 2012), Österreich (Schildberger et al. 2017) und Polen (Mazurkiewicz et al. 2018). Zugangsbarrieren und erfahrene Stigmatisierung und Diskriminierung stehen im Zentrum der Ergebnisse dieser Studien. Autonomieverlust geht dabei häufig mit einem Gefühl einher, kontrolliert und überwacht zu werden. Viele Befragte berichteten von einem Mangel an Unterstützung und Vertrauen der Fachkräfte in ihre Kapazität, die Aufgabe der Elternschaft zu bewältigen. Bradbury-Jones et al. (2015) berichten z. B. von Interviews mit fünf Frauen* mit Behinderung, in denen insgesamt 45 kritische Vorfälle aufgedeckt wurden, die im Zusammenhang mit der Inanspruchnahme von perinatalen Gesundheitsleistungen stehen. Dazu gehören z. B. fehlende oder falsche Informationsvermittlung, mangelnde Einbindung in Entscheidungsprozesse und Missachtung bis hin zur Verweigerung von Behandlungsentscheidungen durch das Fachpersonal.

Auch über die perinatalen Erfahrungen im Gesundheitswesen von Menschen, die sich im LGBTIQ+ Spektrum verorten, ist in Europa wenig bekannt. Von den acht in der Literatursuche identifizierten Studien wurden sechs in Skandinavien (Larsson und Dykes 2009; Röndahl et al. 2009; Dahl et al. 2013; Dahl und Malterud 2015; Wells und Lang 2016; Appelgren Engström et al. 2018) und zwei in Großbritannien (Wilton und Kaufmann 2001; Cherguit et al. 2013) durchgeführt und behandeln alle die Erfahrungen von lesbischen Frauen* mit perinataler Gesundheitsversorgung. Diese Studien zeigen den internalisierten Heterosexismus vieler Fachkräfte in einer auf Heteronormativität ausgerichteten Gesellschaft. Lesbische Paare sehen sich darin mit Diskriminierung aufgrund heterozentristischer struktureller Barrieren, heteronormativer Kommunikation und Vorurteilen konfrontiert. GBTIQ+ Erfahrungen sind in der qualitativen Gesundheitsforschung im Kontext der perinatalen Versorgung keine zu finden. Wells und Lang (2016) haben diese in ihrer systematischen Review zwar einschließen wollen, zeigten aber die Leerstelle in der Forschung auf und mussten sich deshalb in ihrer Analyse auf lesbische Erfahrungen im Themenfeld fokussieren. Trans* und queere Perspektiven auf Schwangerschaft und Elternschaft erscheinen in Deutschland als eine Neuheit in der Forschung. So beginnen im deutschsprachigen Raum zunächst die Sozialwissenschaften ein Interesse zu zeigen (Spahn 2017; Weber 2018; Rewald 2019; Peukert et al. 2020). 


\section{Diskussion}

Perinatale Ungleichheiten bestehen; unabhängig von existierenden Studienergebnissen und Zahlen. Ungerechtigkeit und Ungleichheit in der Betreuung lassen sich schlecht in Zahlen bemessen, wenn nicht diverse potenzielle Faktoren wie Armut oder Reichtum, Geschlechtsidentifizierung, Herkunft, Herkunftsidentifikation, körperliche Beeinträchtigung, Lernschwierigkeiten und dergleichen in ihrer spezifischen Verwobenheit ausreichend berücksichtig werden. Zufriedenstellende Publikationen sowohl qualitativer als auch quantitativer Ergebnisse stehen aus. Qualitative Forschung zu Erfahrungen einzelner Gruppen mit perinataler Betreuung und der daraus resultierenden Zufriedenheit sowie Diskriminierungserfahrungen jeglicher Art gibt es nicht oder nur sehr unzureichend.

Erste bekannte unethische Forschung im Kontext von Gynäkologie und Geburtshilfe in den USA beschreibt z. B. wie James M. Sims experimentelle Operationen an seinen Schwarzen Sklavinnen durchgeführt hat, um vermeintlichen medizinischen Fortschritt für wohlhabende weiße Menschen zu erlangen (Washington 2006). In Deutschland ist dergleichen nur sehr wenig bekannt. Es ist davon auszugehen, dass Menschenforschung in der Weimarer Republik vor allem an sozial schwachen und armen Menschen durchgeführt wurde. Allerdings verweist Reuland (2017) darauf, dass dieser Zusammenhang nur schwer zu belegen ist. Selbst im Nationalsozialismus, der bekannt ist für medizinische Experimente an Menschen, die nicht in das „gesunde arische Rassenkonzept“ passten, ist im Verhältnis wenig über geburtshilfliche Forschung bekannt (Jütte et al. 2012; Schwartz 2014).

Aus einem feministischen Ungerechtigkeitsempfinden heraus entstand in den USA das ABM, bemüht um eine selbstbestimmte und respektvolle Geburtshilfe. Aus Mangel an Anerkennung ethnischer Minderheiten, Schwarzer und sozial Benachteiligter sowie anderer Minoritäten entwickelte sich das BJM. Ähnliche Umstände sind auch in Deutschland zu beobachten. In den 1980er-Jahren wurden in Deutschland in Anlehnung an das ABM die ersten Geburtshäuser gegründet (Netzwerk Geburtshäuser 2021). Geburtsaktivist*innen streiten heute wieder vermehrt für eben jene Ziele. Allerdings wurden auch in Deutschland Minderheiten samt ihren Belangen zunächst nicht oder nicht ausreichend bedacht und sind nach wie vor in Aktivist*innengruppen kaum vertreten. Auch die Deutsche Gesellschaft für Hebammenwissenschaft beschäftigt sich seit einiger Zeit mit dem Thema Respekt und Gewalt in der Geburtshilfe und veröffentlichte 2020 ein Positionspapier dazu (Limmer et al. 2020). Intersektionale Perspektiven wurden jedoch auch in diesem Positionspapier noch nicht mitgedacht.

Anhand der in Deutschland erhobenen Perinataldaten könnte man allgemein schlussfolgern, dass perinatale Ungleichheiten vor allem sozial benachteiligte Menschen betreffen, unabhängig von Herkunft, Abstammung und Migration. Diese Behauptung verhindert jedoch die Berücksichtigung individueller Unterschiede in Forschung und Praxis. Die Summe vielfältiger Identitätsmerkmale sowie biopsychosoziale Prozesse bedingen allgemein unterschiedliche Voraussetzungen für Gesundheit und damit den Verlauf von Schwangerschaft, Geburt und Wochenbett (Scott et al. 2019). So kann die Abstammung z.B. die Geburtsdauer beeinflussen (Greenberg et al. 2006). Sind die Betreuenden sich dessen jedoch nicht bewusst und berück- 
sichtigen dies folglich nicht ausreichend, kann dies dazu führen, dass Gebärende unnötige Interventionen erleben, die nicht nur das Outcome ihrer Geburt beeinflussen, sondern auch ihr Erlebnis derselben und ihren psychischen Umgang damit (Hollins Martin und Fleming 2011; Feinberg et al. 2016).

Generell sagen quantitative Daten, die die Häufigkeit von Untersuchungen bestimmen, wenig über deren Qualität aus. So hat das Robert-Koch-Institut (RKI 2020) festgestellt, dass Schwangere mit sogenanntem Migrationshintergrund im Verhältnis deutlich häufiger ärztliche Vorsorgeuntersuchungen in Anspruch nehmen. Sie stellten aber auch fest, dass diese deutlich seltener Geburtsvorbereitungskurse besuchen und wesentlich seltener kontinuierlich von Hebammen über den gesamten Zeitraum betreut werden. Geburtsvorbereitung hat das Potenzial eines positiven Effekts auf Schwangerschaft, Geburt und Wochenbett (Ferguson et al. 2013). Ergebnisse einer amerikanischen Studie belegen, dass Frauen* in Hebammenbetreuung sowohl deutlich bessere Outcomes aufzeigen als auch geringere intrauterine Mortalität im Vergleich zu jenen in ärztlicher Betreuung (MacDorman und Singh 1998). Darüber hinaus verlaufen hebammengeleitete Geburten deutlich interventionsärmer (Souter et al. 2019) und tragen somit zu maternaler* und kindlicher Gesundheit bei. Diese Ergebnisse zusammengenommen weisen sehr wohl auf migrationsbedingte und/ oder rassifizierte Ungerechtigkeit hin, die nicht in Zahlen messbar ist. Während gender-non-konforme werdende Eltern zwangsläufig aufgrund alltäglicher Diskriminierungserfahrungen reflektiert ihre perinatale Betreuung wählen müssen, bleibt Migrant*innen und sozial benachteiligten Eltern dieser alternative Zugang meist verschlossen aufgrund mangelnder oder zu später Informationsvermittlung.

Das spezifische Zusammenwirken von Rassifizierung, Bildungsstand und finanzieller Abhängigkeit kann bewirken, dass die finanziellen Mittel schließlich die Inanspruchnahme bestimmter Leistungen verhindern können, selbst wenn werdende Eltern das notwendige Wissen und Zugang zu Hebammenbetreuung haben. All diese Faktoren zusammen führen zu Erfahrungen und Anekdoten im Alltag von Hebammen und Eltern, die bisher in Deutschland nicht wissenschaftlich untersucht wurden und daher unter dem Aspekt der Gerechtigkeit nicht erwähnt werden.

Die Faktoren, die den Zugang und die Inanspruchnahme von Gesundheitsleistungen beeinflussen, bedingen sich zyklisch, d. h. die Zufriedenheit der Klient*innen mit der erhaltenen Dienstleistung sind Teil der Faktoren, die zukünftige Gesundheitsentscheidungen beeinflussen. Je positiver die Erfahrungen der Klient*innen sind, desto wahrscheinlicher ist es, dass sie erneut (frühen) Kontakt mit Gesundheitsdienstleister*innen aufnehmen (Bradbury-Jones et al. 2015). Strukturelle Diskriminierungserfahrungen im Gesundheitswesen führen daher zu einer mangelnden Zufriedenheit mit der Betreuung, wirken gleichzeitig in Zukunft als Barriere und perpetuieren damit gesundheitliche Ungleichheit.

Ohne Zweifel braucht es eine regelmäßige und systematische Evaluierung der Erfahrungen mit der perinatalen Versorgung auf nationaler Ebene (Limmer et al. 2020). Systematische, repräsentative Daten gibt es in diesem Kontext im deutschsprachigen Raum bisher nicht. Diskriminierungserfahrungen in der Geburtshilfe wurden in Deutschland bisher nicht im Speziellen untersucht. Um Erfahrungen differenziert zu analysieren, benötigt es Antidiskriminierungskategorien, die über die Erfassung eines sogenannten Migrationshintergrundes hinausgehen. Wichtig sind in 
diesem Zusammenhang sowohl Selbstbezeichnungen als auch zugeschriebene Identitäten innerhalb der Differenzierungsmerkmale und -Dimensionen, um aufzeigen zu können, inwieweit sich spezifische Erfahrungen wiederholen und somit strukturelle Probleme darstellen (Ahyoud et al. 2018).

Die quantitativen Erhebungen in Deutschland sind vor dem beschriebenen Hintergrund vehement zu kritisieren, da eine Unterscheidung der Daten in Populationen mit und ohne Migrationshintergrund nicht differenziert genug sind, um aussagkräftige Schlüsse ziehen zu können. Da entsprechende Studien aus den USA nahelegen, dass sich Stress aufgrund von Diskriminierungserfahrungen deutlich auf gesundheitliche Parameter auswirkt (Krieger 2014), sollten auch hiesige epidemiologische Studien auf der Grundlage differenzierter Antidiskriminierungsdaten durchgeführt werden.

Qualitative Forschungen im Themenfeld Geburtshilfe, Gerechtigkeit und Diskriminierung sind dringend notwendig, um Versorgungsdefizite identifizieren zu können, spezifische Interaktions- und Kommunikationsformen zwischen Gesundheitsfachpersonal und Klient*innen zu analysieren und wahrgenommene Diskriminierungssituationen und Zuschreibungsprozesse aus der Subjektperspektive sichtbar zu machen (Ohlbrecht 2016). Bisher stellen qualitative Untersuchungen zu sozial ungleichen Gesundheitschancen sowie zur ungleichen Versorgungsqualität speziell marginalisierter Gruppen eine Forschungslücke dar. Auch Studien mit partizipativen Designs stehen im Kontext der perinatalen Gesundheitsversorgung im deutschsprachigen Raum noch aus und haben eine besondere Bedeutung, um die Betroffenen aktiv in die Forschung einbinden zu können (Franck et al. 2020). Um Studien zu entwickeln, die den komplexen Lebensrealitäten der Befragten entsprechen, und um zu verhindern, dass unterschiedliche Diskriminierungsformen reproduziert werden, gilt es für Forschende, immer wieder die eigene Haltung und alle Schritte des Forschungsprozesses kritisch und diskriminierungssensibel zu reflektieren (Voß 2019). Schließlich scheinen auch Mixed-Methods-Ansätze sinnvoll, um quantitative Daten mit individuellen Erfahrungen zu kombinieren und somit ein tieferes Verständnis komplexer Zusammenhänge zu erlangen (Peterwerth und Schäfers 2020).

Die Betreuung von Personen, die von Normen der Dominanzgesellschaft abweichen, erfordert ein erhebliches Maß an Reflektion, Offenheit, Flexibilität, Respekt und Empathie. Eine individuelle Beziehungsarbeit zwischen Betreuenden und Betreuten geht vor dem Hintergrund der neoliberalen Ökonomisierung der Geburtshilfe allzu oft verloren (Jung 2017). Kulturell-sensible Betreuung ist zweifelsohne wichtig. Darüber hinaus ist jedoch die Befähigung des Fachpersonals im Gesundheitswesen zu rassismussensibler und -reflektierender bzw. antirassistischer und traumasensibler Betreuung von essenzieller Bedeutung. Dies gilt selbstverständlich auch für Forschende im Themenfeld. Sensibilisierung von Fachkräften im Gesundheitswesen mit Hinblick auf die Lebenssituationen diverser Lebensweisen sind dringend notwendig.

\subsection{Stärken und Schwächen}

Der Beitrag zeigt einen kritischen Umgang mit dem Thema Gerechtigkeit und Diskriminierung im Kontext der peripartalen Gesundheitsversorgung, wodurch eine 
neue intersektionale Perspektive für gesundheitswissenschaftliche Forschung und Versorgungspraxis eröffnet wird. Es werden nicht nur die Grenzen existierender Studien aufgezeigt, sondern auch Anregungen für zukünftige Forschung gegeben, um die gelebten Erfahrungen von marginalisierten und minorisierten Schwangeren und Gebärenden erfassen und damit strukturelle Probleme adressieren zu können.

Der Beitrag zeigt Schwächen in den Recherchemethoden. Systematischere Literaturrecherchen und Analysen hätten eventuell mehr Studien identifiziert. Da aber die Bewertung dieser verhältnismäßig ähnlich war, wurde in diesem Rahmen darauf verzichtet, in der Annahme, dass mehr Studien nicht notwendigerweise neue und/ oder bessere Erkenntnisse gewährt hätten.

\section{Schlussfolgerung}

Abschließend ist festzuhalten, dass kaum deutschsprachige Studien zu Gewalt- und Diskriminierungserfahrungen im Kontext von Schwangerschaft, Geburt und früher Elternschaft im Allgemeinen sowie intersektionale Studien im Besonderen publiziert sind. Um menschenrechtsbasierte, respektvolle Betreuung als Qualitätsmerkmal der Gesundheitsversorgung Realität werden zu lassen und gesundheitliche Ungleichheiten abzubauen, braucht es dringend verlässliche Erhebungen zu Erfahrungen mit der peripartalen Versorgung insbesondere von marginalisierten und minorisierten Personen. Diese sollten in ihrem Design intersektional reflektiert und diskriminierungssensibel angelegt sein. In diesem Sinne sollten unterschiedliche Diskriminierungsformen möglichst umfangreich erfasst sowie ihr Zusammenspiel und ihre Verwobenheit ergründet werden, um strukturelle Probleme adressieren zu können. Bereits im Planungsprozess sollte die eigene Haltung der Forschenden achtsam reflektiert werden, um zu verhindern, dass die eigene Forschung Diskriminierung reproduziert. Weiterhin erscheint die Einbindung von potenziell Betroffenen bereits in die Entwicklung des Studiendesigns essenziell, um spezifische Interessen, Bedürfnisse und Problemlagen adäquat zu erkunden. Gut geplante, intersektional orientierte Studien, durchgeführt von einem möglichst interprofessionellen Team ${ }^{7}$ bergen das Potenzial multiperspektivischer Analysen der geburtshilflichen kulturellen Praxis und können damit einen Beitrag zum Abbau peripartaler Ungerechtigkeit leisten. Dahingehend stellen konzeptionelle und methodologische Überlegungen für intersektionale gesundheitswissenschaftliche Forschung das dringendste Forschungsdesiderat dar.

Auch wenn es einzelne Fachkräfte in der gesundheitlichen Versorgung und Begleitung schwangerer und gebärender Personen gibt, die bereits achtsam und diskriminierungssensibel praktizieren, braucht es diese Forschung, um eine solche Versorgungspraxis als anerkannte Norm in einem von struktureller Diskriminierung geprägten geburtshilflichen System zu etablieren. Die Aus-, Fort- und Weiterbildung aller mittelbar und unmittelbar in der Beratung und gesundheitlichen Versorgung/ Begleitung tätigen Fachkräfte sollte solche Forschungserkenntnisse berücksichtigen und sie zur Grundlage der beruflichen Praxis machen.

\footnotetext{
7 Z. B. Hebammenwissenschaftler*innen, Gynäkolog*innen, Soziolog*innen, Psycholog*innen und ande-
} re Sozial- und Gesundheitswissenschafler*innen sowie Schwangere/Gebärende und deren Angehörige. 
Nichtsdestotrotz ist eine Sensibilisierung für die individuellen Lebensrealitäten derjenigen, die Diskriminierung erfahren, umgehend geboten. Jedes einzelne im Kontext von Schwangerschaft, Geburt und früher Elternschaft praktizierende Individuum kann auch ohne wissenschaftliche Erkenntnisse bereits jetzt durch entsprechende Aus-, Fort- und Weiterbildung zu einer positiven Veränderung der Erfahrung von betroffenen (werdenden) Eltern beitragen. Damit können alle Beteiligten sofort zu mehr Zufriedenheit beitragen und so die Gesundheit von vulnerablen Familien fördern.

Intersektional orientierte Forschung und Praxis im Dienst einer gerechten Versorgung und Begleitung während Schwangerschaft, Geburt und früher Elternschaft kann damit einen Beitrag zum Abbau perinataler Ungerechtigkeit leisten.

Funding Open Access funding enabled and organized by Projekt DEAL.

Open Access Dieser Artikel wird unter der Creative Commons Namensnennung 4.0 International Lizenz veröffentlicht, welche die Nutzung, Vervielfältigung, Bearbeitung, Verbreitung und Wiedergabe in jeglichem Medium und Format erlaubt, sofern Sie den/die ursprünglichen Autor(en) und die Quelle ordnungsgemäß nennen, einen Link zur Creative Commons Lizenz beifügen und angeben, ob Änderungen vorgenommen wurden.

Die in diesem Artikel enthaltenen Bilder und sonstiges Drittmaterial unterliegen ebenfalls der genannten Creative Commons Lizenz, sofern sich aus der Abbildungslegende nichts anderes ergibt. Sofern das betreffende Material nicht unter der genannten Creative Commons Lizenz steht und die betreffende Handlung nicht nach gesetzlichen Vorschriften erlaubt ist, ist für die oben aufgeführten Weiterverwendungen des Materials die Einwilligung des jeweiligen Rechteinhabers einzuholen.

Weitere Details zur Lizenz entnehmen Sie bitte der Lizenzinformation auf http://creativecommons.org/ licenses/by/4.0/deed.de.

Interessenkonflikt C. Winkler und E. Babac geben an, dass kein Interessenkonflikt besteht.

\section{Literatur}

\section{Verwendete Literatur}

Ahrne, Malin, Erica Schytt, Ewa Andersson, Rhonda Small, Aisha Adan, Brigitta Essén, und Ulrika Byrskog. 2019. Antenatal care for Somali-born women in Sweden: perspectives from mothers, fathers and midwives. Midwifery 74:107-115. https://doi.org/10.1016/j.midw.2019.03.022.

Ahyoud, Nasiha, Joshua K. Aikins, Samera Bartsch, Naomi Bechert, Daniel Gyamerah, und Lucienne Wagner. 2018. Wer nicht gezählt wird, zählt nicht. Antidiskriminierungs- und Gleichstellungsdaten in der Einwanderungsgesellschaft - eine anwendungsorientierte Einführung. Vielfalt entscheidet - Diversity in Leadership. Citizens for Europe. Berlin. https:/www.kiwit.org/media/material-downloads/ antidiskriminierungs___gleichstellungsdaten_-_einfuehrung.pdf. Zugegriffen: 5. Apr. 2021.

Altman, Molly R., Talita Oseguera, Monica R. McLemore, Ira Kantrowitz-Gordon, und Linda S. Franck. 2019. Information and power: Women of color's experiences interacting with health care providers in pregnancy and birth. Social Science \& Medicine 238:112491. https://doi.org/10.1016/j.socscimed. 2019.112491.

Appelgren Engström, Heléne, Elisabet Häggström-Nordin, Catrin Borneskog, und Anna-Lena Almqvist. 2018. Mothers in same-sex relationships describe the process of forming a family as a stressful journey in a heteronormative world: a Swedish grounded theory study. Maternal and child health journal 22(10):1444-1450. https://doi.org/10.1007/s10995-018-2525-y.

Asian Communities for Reproductive Justice (ACRJ). 2005. A new vision for advancing our movement for reproductive health, reproductive rights and reproductive justice. https://forwardtogether.org/wpcontent/uploads/2017/12/ACRJ-A-New-Vision.pdf. Zugegriffen: 10. Febr. 2021. 
Barskanmaz, Cengiz. 2019. Recht und Rassismus: Das menschenrechtliche Verbot der Diskriminierung aufgrund der Rasse. Berlin: Springer.

Berger, Claudia. 2012. Soziale Aspekte der Frühgeburtlichkeit unter besonderer Berücksichtigung von Schwangeren mit Migrationshintergrund. https://repositorium.ub.uni-osnabrueck.de/handle/urn:nbn: de:gbv:700-2012101610365. Zugegriffen: 10. Febr. 2021.

Black Mamas Matter Alliance, und Center for Reproductive Rights. 2016. Research overview of maternal mortality and morbidity in the United States. https://www.reproductiverights.org/sites/crr. civicactions.net/files/documents/USPA_MH_TO_ResearchBrief_Final_5.16.pdf. Zugegriffen: 10. Febr. 2021.

Bohren, Meghan A., Joshua P. Vogel, Erin C. Hunter, Olha Lutsiv, Supritha K. Makh, A. Metin Gülmezoglu, et al. 2015. The mistreatment of women during childbirth in health facilities globally: a mixed-methods systematic review. PLoS Medicine 12(6):e1001847. https://doi.org/10.1371/journal. pmed.1001847.

Bollini, Paola, Urszula Stotzer, und Philippe Wanner. 2007. Pregnancy outcomes and migration in Switzerland: results from a focus group study. International journal of public health 52(2):78-86. https:// doi.org/10.1007/s00038-007-6003-3.

Boxall, Nicole, Matthias David, Elisabeth Schalinski, Jürgen Breckenkamp, Oliver Razum, und Lars Hellmeyer. 2018. Perinatal outcome in women with a Vietnamese migration background-retrospective comparative data analysis of 3000 deliveries. Geburtshilfe und Frauenheilkunde 78(7):697-706. https://doi.org/10.1055/a-0636-4224.

Bradbury-Jones, Caroline, Julie Taylor, Jenna Breckenridge, John Devaney, Thilo Kroll, und Anne Lazenbatt. 2015. Disabled women's experiences of accessing and utilising maternity services when they are affected by domestic abuse: a critical incident technique study. BMC Pregnancy Childbirth 15:181. https://doi.org/10.1186/s12884-015-0616-y.

Canty, Lucinda. 2020. It's not always rainbows and unicorns: The lived experience of severe maternal morbidity among black women. Doctoral Dissertations. 2426. University of Connecticut. https:// opencommons.uconn.edu/dissertations/2426. Zugegriffen: 15. März 2021.

Carlsson, Tommy, Banaz Balbas, und Elisabet Mattsson. 2019. Written narratives from immigrants following a prenatal diagnosis: qualitative exploratory study. BMC pregnancy and childbirth 19(1):1-7. https://doi.org/10.1186/s12884-019-2292-9.

Center for American Progress. 2018. Exploring African Americans' high maternal and infant death rates. https://cdn.americanprogress.org/content/uploads/2018/01/29114454/012918_

MaternalInfantMortalityRacialDisparities-brief.pdf. Zugegriffen: 10. Febr. 2021.

Centers for Disease Control and Prevention (CDC). 2016. Health United states. Table 10. https://www.cdc. gov/nchs/hus/contents2016.htm. Zugegriffen: 5. Apr. 2021.

Cherguit, Jasmina, Jan Burns, Sharon Pettle, und Fiona Tasker. 2013. Lesbian co-mothers' experiences of maternity healthcare services. Journal of Advanced Nursing 69(6):1269-1278. https://doi.org/10. 1111/j.1365-2648.2012.06115.x.

Creanga, Andreea A., Carla Syverson, Krisit Seed, und William M. Callaghan. 2017. Pregnancy-related mortality in the United States, 2011-2013. Obstetrics \& Gynecology 130(2):366-373. https://doi.org/ 10.1097/AOG.0000000000002114.

Czollek, Leah Carola, Gudrun Perko, Corinne Kaszner, und Max Czollek. 2019. Praxishandbuch Social Justice und Diversity. Theorien, Training, Methoden, Übungen. Weinheim: Beltz Juventa.

Dahl, Bente, und Kirsti Malterud. 2015. Neither father nor biological mother. A qualitative study about lesbian co-mothers' maternity care experiences. Sexual \& Reproductive Healthcare 6(3):169-173. https://doi.org/10.1016/j.srhc.2015.02.002.

Dahl, Bente, Anne M. Fylkesnes, Venke Sørlie, und Kirsti Malterud. 2013. Lesbian women's experiences with healthcare providers in the birthing context: a meta-ethnography. Midwifery 29(6):674-681. https://doi.org/10.1016/j.midw.2012.06.008.

David, M. Charite Berlin (Hrsg.). 2014. Viele Risiken - gutes Outcome? Geburtshilfliche Ergebnisse bei Migrantinnen. https://www.eine-chance-fuer-kinder.de/wp-content/uploads/2014/05/Viele-Risikenund-gutes-Outcome-geburtshilfl.-Ergebnisse-bei-Migrantinnen-Prof.-Dr.-David.pdf. Zugegriffen: 05. Apr. 2021

David, Matthias, Jürgen Pachaly, und Klaus Vetter. 2006. Perinatal outcome in Berlin (Germany) among immigrants from Turkey. Archives of Gynecology and Obstetrics 274(5):271-278. https://doi.org/10. 1007/s00404-006-0182-7.

David, Matthias, Theda Borde, Silke Brenne, Babette Ramsauer, Wolfgang Henrich, Oliver Razum, et al. 2017. Obstetric and perinatal outcomes among immigrant and non-immigrant women in Berlin, 
Germany. Archives of Gynecology and Obstetrics 296(4):745-762. https://doi.org/10.1007/s00404017-4450-5.

David, Matthias, Katrin A. Scherer, Wolfgang Henrich, und Jürgen Breckenkamp. 2018. Does an immigrant background affect the indication, incidence or outcome of emergency cesarean section? Results of the prospective data collection of 111 births. Geburtshilfe und Frauenheilkunde 78(2):167-172. https://doi.org/10.1055/s-0044-100147.

Degrie, Liesbet, Bernadette Dierckx de Casterlé, Chris Gastmans, und Yvonne Denier. 2020. 'Can you please hold my hand too, not only my breast?' The experiences of Muslim women from Turkish and Moroccan descent giving birth in maternity wards in Belgium. PloS one 15(7):e236008. https://doi. org/10.1371/journal.pone.0236008.

von Elm, Erik, Gerhard Schreiber, und Claudia Cornelia Haupt. 2019. Methodische Anleitung für Scoping Reviews (JBI-Methodologie). Evidenz in der Gesundheitsversorgung 143:1-7. https://doi.org/ 10.1016/j.zefq.2019.05.004.

Fair, Frankie, Liselotte Raben, Helen Watson, Victoria Vivilaki, Maria van den Muijsenbergh, Hora Soltani, und ORAMMA team. 2020. Migrant women's experiences of pregnancy, childbirth and maternity care in European countries: a systematic review. PloS one 15(2):e228378. https://doi.org/10.1371/ journal.pone.0228378.

Feinberg, Marc E., E. Jones Damon, Michael E. Roettger, Michelle L. Hostetler, Kari-Lyn Sakuma, Ian M. Paul, und Deborah B. Ehrenthal. 2016. Preventive effects on birth outcomes: buffering impact of maternal stress, depression, and anxiety. Maternal and Child Health Journal 20(1):56-65. https://doi. org/10.1007/s10995-015-1801-3.

Firdous, Tasneema, Zoe Darwin, und Shaima M. Hassan. 2020. Muslim women's experiences of maternity services in the UK: qualitative systematic review and thematic synthesis. BMC pregnancy and childbirth 20(1):1-10. https://doi.org/10.1186/s12884-020-2811-8.

Franck, Linda S., Monica R. McLemore, Shanell Williams, Kathryn Millar, Anastasia Y. Gordon, Williams Shyneida, Larry Rand, et al. 2020. Research priorities of women at risk for preterm birth: findings and a call to action. BMC pregnancy and childbirth 20(1):1-17. https://doi.org/10.1186/s12884019-2664-1.

Garnweidner-Holme, Lisa M., Mirjam Lukasse, Miriam Solheim, und Lena Henriksen. 2017. Talking about intimate partner violence in multi-cultural antenatal care: a qualitative study of pregnant women's advice for better communication in South-East Norway. BMC pregnancy and childbirth 17(1):1-10. https://doi.org/10.1186/s12884-017-1308-6.

Grant, Maria J., und Andrew Booth. 2009. A typology of reviews: an analysis of 14 review types and associated methodologies. Health Information \& Libraries Journal 26:91-108. https://doi.org/10. 1111/j.1471-1842.2009.00848.x.

Greenberg, Mara B., W. Cheng Yvonne, Linda M. Hopkins, Naomi E. Stotland, Allison S. Bryant, und Aaron B. Caughey. 2006. Are there ethnic differences in the length of labor? American journal of obstetrics and gynecology 195(3):743-748. https://doi.org/10.1016/j.ajog.2006.06.016.

Hall, Jenny, Vanora Hundley, Bethan Collins, und Jilian Ireland. 2018. Dignity and respect during pregnancy and childbirth: a survey of the experience of disabled women. BMC pregnancy and childbirth 18(1):1-13. https://doi.org/10.1186/s12884-018-1950-7.

von Hofen-Hohloch, Judith. 2015. Der Einfluss von Migrationshintergrund auf die Geburt. Vergleich von Perinataldaten von Frauen mit und ohne Migrationshintergrund. https://refubium.fu-berlin. de/bitstream/handle/fub188/9641/Diss_JvHofenHohloch_elektronischeVersion.pdf?sequence=1\& isAllowed=y. Zugegriffen: 10. Febr. 2021.

Hoffkling, Alexis, Juno Obedin-Maliver, und Jae Sevelius. 2017. From erasure to opportunity: a qualitative study of the experiences of transgender men around pregnancy and recommendations for providers. BMC Pregnancy Childbirth 17:332. https://doi.org/10.1186/s12884-017-1491-5.

Hollins Martin, Caroline, und Valerie Fleming. 2011. The birth satisfaction scale. International Journal of Health Care Quality Assurance 24(2):124-135. https://doi.org/10.1108/09526861111105086.

Janevic, Teresa, Pooja Sripad, Elizabeth Bradley, und Vera Dimitrievska. 2011. „There's no kind of respect here". A qualitative study of racism and access to maternal health care among Romani women in the Balkans. International Journal for Equity in Health 10(1):1-12. https://doi.org/10.1186/1475-927610-53.

Jung, Tina. 2017. Die ,gute Geburt“ - Ergebnis richtiger Entscheidungen? Zur Kritik des gegenwärtigen Selbstbestimmungsdiskurses vor dem Hintergrund der Ökonomisierung des Geburtshilfesystems. GENDER - Zeitschrift für Geschlecht, Kultur und Gesellschaft 9(2):9-10.

Jütte, Robert, Wolfgang U. Eckart, und Hans-Walter Schmuhl. 2012. Medizin und Nationalsozialismus: Bilanz und Perspektiven der Forschung. Göttingen: Wallstein. 
Kelly, Natasha A. (Hrsg.). 2019. Schwarzer Feminismus. Grundlagentexte. Münster: Unrast.

Kitchen Politics (Hrsg.). 2021. Mehr als Selbstbestimmung! Kämpfe für reproduktive Gerechtigkeit. Münster: edition assemblage.

Krieger, Nancy. 2014. Discrimination and health inequities. International Journal of Health Services 44(4):643-710.

Larsson, Anna-Karin, und Anna-Karin Dykes. 2009. Care during pregnancy and childbirth in Sweden: perspectives of lesbian women. Midwifery 25(6):682-690. https://doi.org/10.1016/j.midw.2007.10. 004.

LeMasters, Katherine, Anne Wallis Baber, Razvan Chereches, Margaret Gichane, Ciprian Tehei, Andreea Varga, und Katherine Tumlinson. 2019. Pregnancy experiences of women in rural Romania: understanding ethnic and socioeconomic disparities. Culture, health \& sexuality 21(3):249-262. https:// doi.org/10.1080/13691058.2018.1464208.

Limmer, Claudia, Sabine Striebich, Dorothea Tegethoff, Tina Jung, Julia Leinweber, und Deutsche Gesellschaft für Hebammenwissenschaft e. V. (DGHWi). 2020. Respektlosigkeit und Gewalt in der Geburtshilfe. Position der Deutschen Gesellschaft für Hebammenwissenschaft e. V. Zeitschrift für Hebammenwissenschaft 8(2):46-48.

Lu, Michael C., und Neal Halfon. 2003. Racial and ethnic disparities in birth outcomes: a life-course perspective. Maternal and Child Health Journal 7:13-30. https://doi.org/10.1023/A:1022537516969.

Lu, Michael C., Milton Kotelchuk, Vijaya Hogan, Loretta Jones, Kynna Wright, und Neil Halfon. 2010. Closing the black-white gap in birth outcomes: a life course approach. Ethnicity \& Disease $20(10$ 2):S2-62-76.

MacDorman, M. F., und Singh, G. K. 1998. Midwifery care, social and medical risk factors, and birth outcomes in the USA. Journal of Epidemiology \& Community Health 52(5): 310-317. https://doi. org/10.1136/jech.52.5.310.

Malouf, Reem, Jenny McLeish, Sara Ryan, Ron Gray, und Maggie Redshaw. 2017. 'We both just wanted to be normal parents': a qualitative study of the experience of maternity care for women with learning disability. BMJ open https://doi.org/10.1136/bmjopen-2016-015526.

Mazurkiewicz, Barbara, Małgorzata Stefaniak, und Ewa Dmoch-Gajzlerska. 2018. Perinatal care needs and expectations of women with low vision or total blindness in Warsaw, Poland. Disability and health journal 11(4):618-623. https://doi.org/10.1016/j.dhjo.2018.05.005.

Mertens, Alina. 2016. Behinderung und reproduktive Selbstbestimmung. In Geschlechtliche, sexuelle und reproduktive Selbstbestimmung. Praxisorientierte Zugänge, Hrsg. Michaela Katzer, Heinz-Jürgen Voß, 315-331. Gießen: Psychosozial Verlag.

Miani, C., A. Ludwig, J. Breckenkamp, O. Sauzet, I.-M. Doyle, O. Razum, et al. 2020. Socioeconomic and migration status as predictors of emergency caesarean section: a birth cohort study. BMC Pregnancy and Childbirth 20(1):32. https://doi.org/10.1186/s12884-020-2725-5.

Monroe, Shafia. 2015. Foreword. In Birthing justice: black women, pregnancy, and childbirth, 1. Aufl., Hrsg. Julia Chinyere Oparah, Alicia D. Bonaparte. New York: Routledge.

Monroe, Shafia M. 2020. Perinatal disparities and solutions. North Carolina Medical Journal 81(1):56-57. https://doi.org/10.18043/ncm.81.1.56.

National Advocates for Pregnant Women (NAPW). 2010. NAPW working paper: birth justice as reproductive justice. January 2010 draft.

National Partnership for Women \& Families. 2018. Black women's maternal health: a multifaceted approach to addressing persisitent and dire health disparities. Washington: National Partnership for Women \& Families. Issue Brief.

Netzwerk der Geburtshäuser. 2021. Chronologie. https://www.netzwerk-geburtshaeuser.de/chronologie/. Zugegriffen: 14. Apr. 2021

Nixon, Laura. 2013. The right to (trans) parent: A reproductive justice approach to reproductive rights, fertility, and family-building issues facing transgender people. William and Mary Journal of Women and Law 20(1):73-103.

Ohlbrecht, Heike. 2016. Die qualitative Analyse von Gesundheit und Krankheit. In Soziologie von Gesundheit und Krankheit, Hrsg. Matthias Richter, Klaus Hurrelmann, 71-87. Wiesbaden: Springer VS.

Oparah, Julia Chinyere. 2015. Beyond coercion and malign neglect. Black women and the struggle for birth justice. In Birthing justice: black women, pregnancy, and childbirth, Hrsg. Julia Chinyere Oparah, Alicia D. Bonaparte, 1-18. New York: Routledge.

Oparah, Julia Chinyere, und Alicia D. Bonaparte (Hrsg.). 2015. Birthing justice: black women, pregnancy, and childbirth. New York: Taylor \& Francis.

Origlia Ikhilor, Paola, Gabriele Hasenberg, Elisabeth Kurth, Fana Asefaw, Jessica Pehlke-Milde, und Eva Cignacco. 2019. Communication barriers in maternity care of allophone migrants: experiences 
of women, healthcare professionals, and intercultural interpreters. Journal of advanced nursing 75(10):2200-2210. https://doi.org/10.1111/jan.14093.

Peterwerth, Nina H., und Reinhild Schäfers. 2020. Potenzial des Mixed-Methods-Ansatzes in der Hebammenwissenschaft am Beispiel einer Studie zur Risikowahrnehmung geburtshilflicher Fachpersonen. Zeitschrift für Hebammenwissenschaft 8(2):62-68.

Peukert, Almut, Julia Teschlade, Christine Wimbauer, Mona Motakef, und Elisabeth Holzleithner (Hrsg.). 2020. Elternschaft und Familie jenseits von Heteronormativität und Zweigeschlechtlichkeit. Opladen: Barbara Budrich.

Price, Kimala. 2020. What is reproductive justice? How women of color activists are redefining the prochoice paradigm. Meridians: feminism, race, transnationalism 19:340-362.

Razum, Oliver, Albrecht Jahn, Maria Blettner, und Pitt Reitmaier. 1999. Trends in maternal mortality ratio among women of German and non-German nationality in West Germany, 1980-1996. International journal of epidemiology 28(5):919-924. https://doi.org/10.1093/ije/28.5.919.

Reeske, Anna, Marcus Kutschmann, Oliver Razum, und Jacob Spallek. 2011. Stillbirth differences according to regions of origin: an analysis of the German perinatal database, 2004-2007. BMC Pregnancy and Childbirth 11:63. https://doi.org/10.1186/1471-2393-11-63.

Reime, Birgit, Pamela A. Ratner, Sandra N. Tomaselli-Reime, Ann Kelly, Beate A. Schuecking, und Paul Wenzlaff. 2006. The role of mediating factors in the association between social deprivation and low birth weight in Germany. Social Science \& Medicine 62(7):1731-1744. https://doi.org/10.1016/j. socscimed.2005.08.017.

Reuland, Andreas. 2017. Menschenversuche in der Weimarer Republik. Heidelberg: heiBOOKS. https:// doi.org/10.11588/heibooks.260.345.

Rewald, Sascha. 2019. Elternschaften von trans Personen. Trans Eltern zwischen rechtlicher Diskriminierung, gesundheitlicher Unterversorgung und alltäglicher Herausforderung. In Trans \& Care. Trans Personen zwischen Selbstsorge, Fürsorge und Versorgung, Hrsg. Max N. Appenroth, Maria do Mar Castro Varela, 187-199. Bielefeld: transcript.

Robert Koch Institut (RKI). 2020. Gesundheitliche Lage der Frauen in Deutschland. Kapitel 6. Gesundheit von Frauen mit Migrationshintergrund, https:/www.rki.de/DE/Content/Gesundheitsmonitoring/ Gesundheitsberichterstattung/GBEDownloadsB/frauenbericht/06_Frauen_mit_Migrationshinter grund.pdf?_blob=publicationFile.

Robertson, Eva K. 2015. "To be taken seriously": women's reflections on how migration and resettlement experiences influence their healthcare needs during childbearing in Sweden. Sexual \& Reproductive Healthcare 6(2):59-65. https://doi.org/10.1016/j.srhc.2014.09.002.

Röndahl, Gerd, Elisabeth Bruhner, und Jenny Lindhe. 2009. Heteronormative communication with lesbian families in antenatal care, childbirth and postnatal care. Journal of Advanced Nursing 65(11):2337-2344. https://doi.org/10.1111/j.1365-2648.2009.05092.x.

Ross, Loretta J., und Rickie Solinger. 2017. Reproductive justice. An introduction. Oakland: University of California Press.

Schildberger, Barbara, Christoph Zenzmaier, und Martina König-Bachmann. 2017. Experiences of Austrian mothers with mobility or sensory impairments during pregnancy, childbirth and the puerperium: a qualitative study. BMC pregnancy and childbirth 17(1):1-11. https://doi.org/10.1186/s12884-0171388-3.

Schmidt, N.C., V. Fargnoli, M. Epiney, und O. Irion. 2018. Barriers to reproductive health care for migrant women in Geneva: a qualitative study. Reproductive health 15(1):1-10. https://doi.org/10.1186/ s12978-018-0478-7.

Schwartz, Michael (Hrsg.). 2014. Homosexuelle im Nationalsozialismus: Neue Forschungsperspektiven zu Lebenssituationen von lesbischen, schwulen, bi-, trans- und intersexuellen Menschen 1933 bis 1945. Bd. 18. Berlin: de Gruyter.

Scott, Karen A., Laura Britton, und Monica R. McLemore. 2019. The ethics of perinatal care for black women: dismantling the structural racism in "mother blame" narratives. The Journal of perinatal \& neonatal nursing 33(2):108-115. https://doi.org/10.1097/JPN.0000000000000394.

Souter, Vivienne, Elizabeth Nethery, Mary Lou Kopas, Hannah Wurz, Kristin Sitcov, und Aaron B. Caughey. 2019. Comparison of Midwifery and Obstetric Care in Low-Risk Hospital Births. Obstetrics \& Gynecology 134(5):1056-1065. https://doi.org/10.1097/AOG.0000000000003521.

Spahn, Annika. 2017. Subversion oder Assimilation? Trans* und Schwangerschaft in einer heteronormativen Gesellschaft. freidok.uni-freiburg.de/data/12314. Zugegriffen: 20. Jan. 2021.

Spallek, J., J. Lehnhardt, A. Reeske, O. Razum, und M. David. 2014. Perinatal outcomes of immigrant women of Turkish, Middle Eastern and North African origin in Berlin, Germany: a comparison of 
two time periods. Archives of Gynecology and Obstetrics 289(3):505-512. https://doi.org/10.1007/ s00404-013-2986-6.

Utne, Renate, Chloe Lindsay Antrobus-Johannessen, Vigdis Aasheim, Katrine Aasekjær, und Eline Skirnisdottir Vik. 2020. Somali women's experiences of antenatal care: a qualitative interview study. Midwifery 83:102656. https://doi.org/10.1016/j.midw.2020.102656.

Voß, Heinz-Jürgen. 2019. Rassismus überwinden: Ein Umdenken in sexualwissenschaftlicher Forschung ist erforderlich. In Rassismus an Hochschulen: Analyse - Kritik - Interventionen, Hrsg. Daniela Heitzmann, Kathrin Houda, 241-258. Weinheim: Beltz Juventa.

Walsh-Gallagher, Dympna, Marlene Sinclair, und Roy McConkey. 2012. The ambiguity of disabled women's experiences of pregnancy, childbirth and motherhood: a phenomenological understanding. Midwifery 28(2):156-162. https://doi.org/10.1016/j.midw.2011.01.003.

Washington, Harriet A. 2006. Medical apartheid. New York: Penguin Randomhouse.

Watson, Helen L., und Soo Downe. 2017. Discrimination against childbearing Romani women in maternity care in Europe: a mixed-methods systematic review. Reproductive health 14(1):1. https://doi.org/10. 1186/2Fs12978-016-0263-4.

Weber, Rix. 2018. Trans* und Elternschaft. Wie trans* Eltern normative Vorstellungen von Familie und Geschlecht verhandeln. Diss. Niedersächsische Staats- und Universitätsbibliothek Göttingen. http:// hdl.handle.net/11858/00-1735-0000-002E-E50C-3. Zugegriffen: 10. März 2021.

Wells, Michael B., und Sarah N. Lang. 2016. Supporting same-sex mothers in the Nordic child health field: a systematic literature review and meta-synthesis of the most gender equal countries. Journal of clinical nursing 25(23-24):3469-3483. https://doi.org/10.1111/jocn.13340.

Wilton, Tamsin, und Tara Kaufmann. 2001. Lesbian mothers' experiences of maternity care in the UK. Midwifery 17(3):203-211. https://doi.org/10.1054/midw.2001.02.

Zolitschka, Kim A., Céline Miani, Jürgen Breckenkamp, Silke Brenne, Theda Borde, Matthias David, und Oliver Razum. 2019. Do social factors and country of origin contribute towards explaining a „Latina paradox" among immigrant women giving birth in Germany? BMC Public Health 19(1):181. https:// doi.org/10.1186/s12889-019-6523-9.

\section{Weiterführende Literatur}

Barkensjö, M., J.T. Greenbrook, J. Rosenlundh, H. Ascher, und H. Elden. 2018. The need for trust and safety inducing encounters: a qualitative exploration of women's experiences of seeking perinatal care when living as undocumented migrants in Sweden. BMC pregnancy and childbirth 18(1):1-17.

Bick, D., L.M. Howard, S. Oram, und C. Zimmerman. 2017. Maternity care for trafficked women: survivor experiences and clinicians' perspectives in the United Kingdom's national health service. PloS one 12(11):e187856.

Carlsson, T., U.M. Marttala, E. Mattsson, und A. Ringnér. 2016. Experiences and preferences of care among Swedish immigrants following a prenatal diagnosis of congenital heart defect in the fetus: a qualitative interview study. BMC pregnancy and childbirth 16(1):1-8.

Chinouya, M.J., und C. Madziva. 2019. Late booking amongst African women in a London borough, England: implications for health promotion. Health promotion international 34(1):123-132.

Coutinho, E., A. Rocha, C. Pereira, A. Silva, J. Duarte, und V. Parreira. 2014. Experiences of motherhood: unmet expectations of immigrant and native mothers, about the Portuguese health system. Atencion primaria 46:140-144.

Cross-Sudworth, F., A. Williams, und S. Herron-Marx. 2011. Maternity services in multi-cultural Britain: using Q methodology to explore the views of first-and second-generation women of Pakistani origin. Midwifery 27(4):458-468.

Crowther, S., und A. Lau. 2019. Migrant polish women overcoming communication challenges in Scottish maternity services: a qualitative descriptive study. Midwifery 72:30-38.

Dayyani, I., H. Terkildsen Maindal, G. Rowlands, und S. Lou. 2019. A qualitative study about the experiences of ethnic minority pregnant women with gestational diabetes. Scandinavian journal of caring sciences 33(3):621-631.

Ferguson, Sally, Deborah Davis, und Jenny Browne. 2013. Does antenatal education affect labour and birth? A structured review of the literature. Women and Birth 26(1):e5-e8.

Finigan, V., und T. Long. 2014. Skin-to-skin contact: multicultural perspectives on birth fluids and birth 'dirt'. International nursing review 61(2):270-277.

Gardner, P.L., P. Bunton, D. Edge, und A. Wittkowski. 2014. The experience of postnatal depression in West African mothers living in the United Kingdom: a qualitative study. Midwifery 30(6):756-763. 
Haddrill, R., G.L. Jones, C.A. Mitchell, und D.O. Anumba. 2014. Understanding delayed access to antenatal care: a qualitative interview study. BMC pregnancy and childbirth 14(1):1-14.

Hassan, S.M., C. Leavey, und J.S. Rooney. 2019. Exploring English speaking Muslim women's firsttime maternity experiences: a qualitative longitudinal interview study. BMC pregnancy and childbirth 19(1):1-10.

Henriksen, L., E. Grimsrud, B. Schei, M. Lukasse, und Bidens Study Group. 2017. Factors related to a negative birth experience-a mixed methods study. Midwifery 51:33-39.

Lauridsen, D.S., P. Sandøe, und L. Holm. 2018. Being targeted as a "severely overweight pregnant woman"-A qualitative interview study. Health Expectations 21(5):878-886.

Leppälä, S., R. Lamminpää, M. Gissler, und K. Vehviläinen-Julkunen. 2020. Humanitarian migrant women's experiences of maternity care in Nordic countries: a systematic integrative review of qualitative research. Midwifery 80:102572.

Lingen-Stallard, A., C. Furber, und T. Lavender. 2016. Testing HIV positive in pregnancy: a phenomenological study of women's experiences. Midwifery 35:31-38.

Lyons, S., S. Currie, und D.M. Smith. 2019. Learning from women with a body mass index (BMI) $\geq$ $30 \mathrm{~kg} / \mathrm{m} 2$ who have breastfed and/or are breastfeeding: a qualitative interview study. Maternal and child health journal 23(5):648-656.

McFadden, A., M.J. Renfrew, und K. Atkin. 2013. Does cultural context make a difference to women's experiences of maternity care? A qualitative study comparing the perspectives of breast-feeding women of Bangladeshi origin and health practitioners. Health Expectations 16(4):e124-e135.

Montgomery, E., C. Pope, und J. Rogers. 2015. The re-enactment of childhood sexual abuse in maternity care: a qualitative study. BMC pregnancy and childbirth $15(1): 1-7$.

Phillimore, J. 2016. Migrant maternity in an era of superdiversity: new migrants' access to, and experience of, antenatal care in the West Midlands, UK. Social Science \& Medicine 148:152-159.

van der Pijl, M.S., M.H. Hollander, T. van der Linden, R. Verweij, L. Holten, E. Kingma, und C.J. Verhoeven. 2020. Left powerless: a qualitative social media content analysis of the Dutch\# breakthesilence campaign on negative and traumatic experiences of labour and birth. PloS one 15(5):e233114.

Sami, J., K.Q. Lötscher, I. Eperon, L. Gonik, B.M. de Tejada, M. Epiney, und N.C. Schmidt. 2019. Giving birth in Switzerland: a qualitative study exploring migrant women's experiences during pregnancy and childbirth in Geneva and Zurich using focus groups. Reproductive health 16(1):1-9. https://doi. org/10.1186/s12978-019-0771-0.

Schroll, A.M., H. Kjærgaard, und J. Midtgaard. 2013. Encountering abuse in health care; lifetime experiences in postnatal women-a qualitative study. BMC pregnancy and childbirth 13(1):1-11.

Straus, L., A. McEwen, und F.M. Hussein. 2009. Somali women's experience of childbirth in the UK: perspectives from Somali health workers. Midwifery 25(2):181-186.

Vangen, S., R.E.B. Johansen, J. Sundby, B. Træen, und B. Stray-Pedersen. 2004. Qualitative study of perinatal care experiences among Somali women and local health care professionals in Norway. European Journal of Obstetrics \& Gynecology and Reproductive Biology 112(1):29-35.

Watson, H., D. Harrop, E. Walton, A. Young, und H. Soltani. 2019. A systematic review of ethnic minority women's experiences of perinatal mental health conditions and services in Europe. PloS one 14(1):e210587.

Wittkowski, A., S. Patel, und J.R. Fox. 2017. The experience of postnatal depression in immigrant mothers living in Western countries: a meta-synthesis. Clinical psychology \& psychotherapy 24(2):411-427.

Christiane Winkler freiberufliche Hebamme, Jg. 1981; 2002-2007 Studium der Historischen Anthropologie und Europäischen Ethnologie an der Albert-Ludwigs-Universität Freiburg i. Br.; 2008-2011 Ausbildung zur Hebamme in Minden; seit 2012 freiberufliche Hebammentätigkeit in gemeinschaftlicher Praxis in Leipzig; seit 2019 Masterstudium der Angewandten Sexualwissenschaft an der Hochschule Merseburg; seit 2021 wissenschaftliche Mitarbeiterin am Institut für Gesundheits- und Pflegewissenschaft der MartinLuther-Universität Halle-Wittenberg; besonderes Interesse für intersektional-feministische Theorien und Forschung (insb. Reproduktive Gerechtigkeit), lebenslaufbegleitende sexuelle Bildung und Beratung, Familienplanung und Interventionspraxis (insb. Beratung zu pränataler Diagnostik, ungewollter Schwangerschaft). 
Emine Babac freiberufliche Hebamme Jg. 1977; 1997-1999 kurzes Studium der Humanmedizin an der Martin-Luther-Universität in Halle/Saale; 2000-2003 Hebammenausbildung in Osnabrück; 2011-2013 BSc Midwifery an der Glasgow Caledonian University, 2016-2021 MSc Advanced Practice am King's College London; seit 2003 freiberufliche Hebamme; 2012-13 Community und Homebirth Midwife in London, seit 2013 externe Lehrkraft für verschiedene Lehreinheiten im Hebammen- und KrankenpflegeStudium an der Evangelischen Hochschule Berlin (EHB); 2017-2019 Integration-, Homebirth und Caseloading Lead in London zur Implementierung der Better-Births-Ziele. Besondere Interessen: Geburtsphysiologie, außerklinische Geburtshilfe, Diversität und Diskrimierungsbewusstsein in der Geburtshilfe. 